\title{
Some families of generalized complete and incomplete elliptic-type integrals
}

\author{
H. M. Srivastava ${ }^{\mathrm{a}, \mathrm{b}, *}$, Rakesh K. Parmar ${ }^{\mathrm{c}}$, Purnima Chopra ${ }^{\mathrm{d}}$ \\ ${ }^{a}$ Department of Mathematics and Statistics, University of Victoria, Victoria, British Columbia V8W 3R4, Canada. \\ ${ }^{b}$ Department of Medical Research, China Medical University Hospital, China Medical University, Taichung 40402, Taiwan, Republic of \\ China. \\ ${ }^{c}$ Department of Mathematics, Government College of Engineering and Technology, Bikaner 334004, Rajasthan, India. \\ ${ }^{d}$ Department of Mathematics, Marudhar Engineering College, Bikaner 334001, Rajasthan, India.
}

Communicated by R. Saadati

\begin{abstract}
Analogous to the recent generalizations of the familiar beta and hypergeometric functions by Lin et al. [S.-D. Lin, H. M. Srivastava, J.-C. Yao, Appl. Math. Inform. Sci., 9 (2015), 1731-1738], the authors introduce and investigate some general families of the elliptic-type integrals for which the usual properties and representations are naturally and simply extended. The object of the present paper is to study these generalizations and their relationships with generalized hypergeometric functions of one, two and three variables. Moreover, the authors establish the Mellin transform formulas and various derivative and integral properties and obtain several relations for special cases in terms of well-known higher transcendental functions and some infinite series representations containing the Meijer G-function, the Whittaker function and the complementary error functions, as well as the Laguerre polynomials and the products thereof. A number of (known or new) special cases and consequences of the main results presented here are also considered. (C)2017 All rights reserved.
\end{abstract}

Keywords: Incomplete and complete elliptic integrals, generalized Beta function, generalized hypergeometric functions, generalized Appell functions, generalized Lauricella functions, Mellin transforms, Whittaker functions, Laguerre polynomials. 2010 MSC: Primary 26A33, 33C65; Secondary 33C75, 78A40, 78A45.

\section{Introduction, definitions and preliminaries}

In Legendre's normal form, the incomplete elliptic integrals $F(\varphi, k), E(\varphi, k)$ and $\Pi\left(\varphi, \alpha^{2}, k\right)$ of the first, second and third kind (with modulus $|k|$ and amplitude $\varphi$ ) are defined by (see, e.g., $[1,3,9,11]$ ),

$$
\begin{aligned}
\mathrm{F}(\varphi, \mathrm{k}):=\int_{0}^{\varphi} \frac{\mathrm{d} \theta}{\sqrt{1-\mathrm{k}^{2} \sin ^{2} \theta}}=\int_{0}^{\sin \varphi} \frac{\mathrm{dt}}{\sqrt{\left(1-\mathrm{t}^{2}\right)\left(1-\mathrm{k}^{2} \mathrm{t}^{2}\right)}} \\
\quad\left(\left|\mathrm{k}^{2}\right|<1, \quad 0 \leqq \varphi \leqq \frac{\pi}{2}\right),
\end{aligned}
$$

\footnotetext{
*Corresponding author

Email addresses: harimsri@math.uvic.ca (H. M. Srivastava), rakeshparmar27@gmail .com (Rakesh K. Parmar), purnimachopra@rediffmail.com (Purnima Chopra)
}

doi:10.22436/jnsa.010.03.25 


$$
\begin{gathered}
E(\varphi, k):=\int_{0}^{\varphi} \sqrt{1-k^{2} \sin ^{2} \theta} d \theta=\int_{0}^{\sin \varphi} \sqrt{\frac{1-k^{2} t^{2}}{1-t^{2}}} d t \\
\left(\left|k^{2}\right|<1, \quad 0 \leqq \varphi \leqq \frac{\pi}{2}\right),
\end{gathered}
$$

and

$$
\begin{aligned}
& \Pi\left(\varphi, \alpha^{2}, k\right):=\int_{0}^{\varphi} \frac{\mathrm{d} \theta}{\left(1-\alpha^{2} \sin ^{2} \theta\right) \sqrt{1-k^{2} \sin ^{2} \theta}} \\
&=\int_{0}^{\sin \varphi} \frac{\mathrm{dt}}{\left(1-\alpha^{2} \mathrm{t}^{2}\right) \sqrt{\left(1-\mathrm{t}^{2}\right)\left(1-\mathrm{k}^{2} \mathrm{t}^{2}\right)}}, \\
&\left(\left|\mathrm{k}^{2}\right|<1, \quad-\infty<\alpha^{2}<\infty, \quad 0 \leqq \varphi \leqq \frac{\pi}{2}\right),
\end{aligned}
$$

respectively. In particular, when

$$
\varphi=\frac{\pi}{2}
$$

the definitions (1.1), (1.2) and (1.3) reduce immediately to the corresponding complete elliptic integrals $\mathrm{K}(\mathrm{k}), \mathrm{E}(\mathrm{k})$ and $\Pi\left(\alpha^{2}, \mathrm{k}\right)$ of the first, second and third kind, which are defined by

$$
\begin{aligned}
K(k) & :=\int_{0}^{\frac{\pi}{2}} \frac{\mathrm{d} \theta}{\sqrt{1-\mathrm{k}^{2} \sin ^{2} \theta}}=\int_{0}^{1} \frac{\mathrm{dt}}{\sqrt{\left(1-\mathrm{t}^{2}\right)\left(1-\mathrm{k}^{2} \mathrm{t}^{2}\right)}}, \quad\left(\left|\mathrm{k}^{2}\right|<1\right), \\
\mathrm{E}(\mathrm{k}) & :=\int_{0}^{\frac{\pi}{2}} \sqrt{1-\mathrm{k}^{2} \sin ^{2} \theta} \mathrm{d} \theta=\int_{0}^{1} \sqrt{\frac{1-\mathrm{k}^{2} \mathrm{t}^{2}}{1-\mathrm{t}^{2}}} \mathrm{dt}, \quad\left(\left|\mathrm{k}^{2}\right|<1\right),
\end{aligned}
$$

and

$$
\begin{aligned}
\Pi\left(\alpha^{2}, \mathrm{k}\right) & :=\int_{0}^{\frac{\pi}{2}} \frac{\mathrm{d} \theta}{\left(1-\alpha^{2} \sin ^{2} \theta\right) \sqrt{1-k^{2} \sin ^{2} \theta}} \\
& =\int_{0}^{1} \frac{\mathrm{dt}}{\left(1-\alpha^{2} \mathrm{t}^{2}\right) \sqrt{\left(1-\mathrm{t}^{2}\right)\left(1-\mathrm{k}^{2} \mathrm{t}^{2}\right)}}, \quad\left(\left|\mathrm{k}^{2}\right|<1, \quad \alpha^{2} \neq 1\right),
\end{aligned}
$$

respectively.

Over five decades ago, Epstein and Hubbell [19] (and, in a sequel, Weiss [36]) studied the following interesting generalization of $\mathrm{K}(\mathrm{k})$ and $\mathrm{E}(\mathrm{k})$, which was encountered in a Legendre polynomials expansion method when applied to certain problems involving computation of the radiation field off-axis from a uniform circular disk radiating according to an arbitrary angular distribution law (see, for details, [7]):

$$
\begin{gathered}
\Omega_{\mathfrak{j}}(\kappa):=\int_{0}^{\pi} \frac{\mathrm{d} \theta}{\left(1-\kappa^{2} \cos \theta\right)^{j+\frac{1}{2}}}, \\
\left(0 \leqq \kappa<1, \quad \mathfrak{j} \in \mathbb{N}_{0}:=\mathbb{N} \cup\{0\}, \quad \mathbb{N}:=\{1,2,3, \cdots\}\right) .
\end{gathered}
$$

Indeed, by comparing the definitions (1.4), (1.5) and (1.7), we have the following relationships:

$$
\Omega_{0}(\kappa)=\frac{k \sqrt{2}}{\kappa} K(k), \quad \text { and } \quad \Omega_{1}(\kappa)=\frac{k \sqrt{2}}{\kappa\left(1-k^{2}\right)} E(k), \quad\left(k^{2}:=\frac{2 \kappa^{2}}{1+k^{2}}\right) .
$$

Motivated by their importance and also by their potential for applications in certain problems in radiation physics, several recent works were devoted exclusively to the study of various interesting generalizations of the elliptic integrals (see $[6,8,10,17,18,21,22,29,30,34,35]$ ). In particular, Lin et al. [22, p. 1178, Eq. (1.12)] and Bushell [8, p. 2, Eq. (2.2)] studied and investigated the following families 
$H(\varphi, k, \gamma)$, and $H(k, \gamma)$ of incomplete elliptic integrals and complete elliptic integrals:

$$
\begin{aligned}
\mathrm{H}(\varphi, k, \gamma):= & \int_{0}^{\varphi}\left(1-\mathrm{k}^{2} \sin ^{2} \theta\right)^{\gamma-\frac{1}{2}} \mathrm{~d} \theta=\int_{0}^{\sin \varphi} \frac{\left(1-\mathrm{k}^{2} \mathrm{t}^{2}\right)^{\gamma-\frac{1}{2}}}{\sqrt{1-\mathrm{t}^{2}}} \mathrm{dt}, \\
& \left(\left|\mathrm{k}^{2}\right|<1, \quad 0 \leqq \varphi \leqq \frac{\pi}{2}, \quad \gamma \in \mathbb{C}\right),
\end{aligned}
$$

and

$$
\begin{gathered}
H(k, \gamma):=\int_{0}^{\frac{\pi}{2}}\left(1-k^{2} \sin ^{2} \theta\right)^{\gamma-\frac{1}{2}} \mathrm{~d} \theta=\int_{0}^{1} \frac{\left(1-\mathrm{k}^{2} \mathrm{t}^{2}\right)^{\gamma-\frac{1}{2}}}{\sqrt{1-\mathrm{t}^{2}}} \mathrm{dt}, \\
\left(\left|\mathrm{k}^{2}\right|<1, \quad \gamma \in \mathbb{C}\right),
\end{gathered}
$$

respectively, so that, obviously, we have

$$
H(k, \gamma):=H\left(\frac{\pi}{2}, k, \gamma\right), \quad H(\varphi, k, 0)=: F(\varphi, k), \quad \text { and } \quad H(\varphi, k, 1)=: E(\varphi, k),
$$

and

$$
H\left(\frac{\pi}{2}, k, 0\right)=: K(k), \quad \text { and } \quad H\left(\frac{\pi}{2}, k, 1\right)=: E(k) .
$$

The literature on Special Functions contains several generalizations of the Gamma function $\Gamma(z)$, the Beta function $B(\alpha, \beta)$, the hypergeometric functions ${ }_{1} F_{1}$ and ${ }_{2} F_{1}$, and the generalized hypergeometric functions ${ }_{r} F_{s}$ with $r$ numerator and $s$ denominator parameters (see, for details, [2, 12-16, 23, 24, 33] and the references cited in each of these papers). In particular, for an appropriately bounded sequence $\left\{\kappa_{\ell}\right\}_{\ell \in \mathbb{N}_{0}}$ of essentially arbitrary (real or complex) numbers, Srivastava et al. [33, p. 243, Eq.(2.1)] recently considered the function $\Theta\left(\left\{\kappa_{\ell}\right\}_{\ell \in \mathbb{N}_{0}} ; z\right)$ given by

$$
\Theta\left(\left\{\kappa_{\ell}\right\}_{\ell \in \mathbb{N}_{0}} ; z\right):= \begin{cases}\sum_{\ell=0}^{\infty} \kappa_{\ell} \frac{z^{\ell}}{\ell !}, & \left(|z|<R, \quad 0<R<\infty, \quad \kappa_{0}:=1\right), \\ \mathfrak{M}_{0} z^{\omega} \exp (z)\left[1+\mathrm{O}\left(\frac{1}{z}\right)\right], & \left(\mathfrak{R}(z) \rightarrow \infty, \quad \mathfrak{M}_{0}>0, \omega \in \mathbb{C}\right),\end{cases}
$$

for some suitable constants $\mathfrak{M}_{0}$ and $\omega$ depending essentially upon the sequence $\left\{\kappa_{\ell}\right\}_{\ell \in \mathbb{N}_{0}}$. In terms of the function $\Theta\left(\left\{\kappa_{\ell}\right\}_{\ell \in \mathbb{N}_{0}} ; z\right)$ defined by (1.8), Srivastava et al. [33] introduced and investigated the following remarkably deep generalizations of the extended Gamma function, the extended Beta function and the extended Gauss hypergeometric function:

$$
\begin{gathered}
\Gamma_{\mathrm{p}}^{\left(\left\{\kappa_{\ell}\right\}_{\ell \in \mathbb{N}_{0}}\right)}(z)=\int_{0}^{\infty} \mathrm{t}^{z-1} \Theta\left(\left\{\kappa_{\ell}\right\}_{\ell \in \mathbb{N}_{0}} ;-t-\frac{p}{t}\right) d t, \\
(\mathfrak{R}(z)>0, \quad \mathfrak{R}(p) \geqq 0), \\
\mathfrak{B}^{\left(\left\{\kappa_{\ell}\right\}_{\ell \in \mathbb{N}_{0}}\right)}(\alpha, \beta ; p):=\int_{0}^{1} t^{\alpha-1}(1-t)^{\beta-1} \Theta\left(\left\{\kappa_{\ell}\right\}_{\ell \in \mathbb{N}_{0}} ;-\frac{p}{t(1-t)}\right) d t, \\
(\min \{\mathfrak{R}(\alpha), \mathfrak{R}(\beta)\}>0, \quad \mathfrak{R}(p) \geqq 0),
\end{gathered}
$$

and

$$
\begin{gathered}
\mathfrak{F}_{\mathfrak{p}}^{\left(\left\{\kappa_{\ell}\right\}_{\ell \in \mathbb{N}_{0}}\right)}(a, b ; c ; z):=\frac{1}{B(b, c-b)} \sum_{n=0}^{\infty}(a)_{n} \mathfrak{B}^{\left(\left\{\kappa_{\ell}\right\}_{\ell \in \mathbb{N}_{0}}\right)}(b+n, c-b ; p) \frac{z^{\mathfrak{n}}}{n !}, \\
(|z|<1, \quad \mathfrak{R}(\mathrm{c})>\mathfrak{R}(b)>0, \quad \mathfrak{R}(\mathrm{p}) \geqq 0),
\end{gathered}
$$

respectively, provided that the defining integrals in the definitions (1.9) and (1.10) exist. 
Lin et al. [23] introduced and investigated a substantially more general family of the generalized Beta function and the Gauss type hypergeometric functions, which are defined by

$$
\begin{aligned}
& \mathfrak{B}_{p ; \mu, v}^{\left(\left\{k_{\ell}\right\}_{\ell \in \mathbb{N}_{0}}\right)}(\alpha, \beta)=\mathfrak{B}^{\left(\left\{\kappa_{\ell}\right\}_{\ell \in \mathbb{N}_{0}}\right)}(\alpha, \beta ; p ; \mu, v) \\
&:=\int_{0}^{1} t^{\alpha-1}(1-t)^{\beta-1} \Theta\left(\left\{\kappa_{\ell}\right\}_{\ell \in \mathbb{N}_{0}} ;-\frac{p}{t^{\mu}(1-t)^{v}}\right) d t, \\
&(\min \{\mathfrak{R}(\alpha), \mathfrak{R}(\beta), \mathfrak{R}(\mu), \mathfrak{R}(v)\}>0, \quad \mathfrak{R}(p) \geqq 0),
\end{aligned}
$$

and

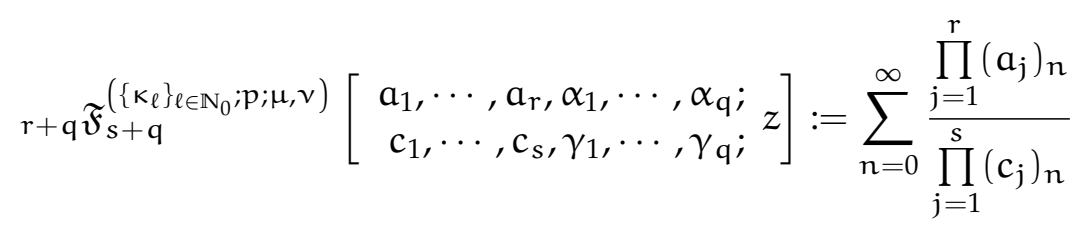

$$
\begin{aligned}
& \cdot \prod_{j=1}^{q} \frac{\mathfrak{B}^{\left(\left\{\kappa_{\ell}\right\}_{\ell \in \mathbb{N}_{0}}\right)}\left(\alpha_{j}+n, \gamma_{j}-\alpha_{j} ; p ; \mu, v\right)}{\mathfrak{B}^{\left(\left\{\kappa_{\ell}\right\}_{\ell \in \mathbb{N}_{0}}\right)}\left(\alpha_{j}, \gamma_{j}-\alpha_{j} ; p ; \mu, v\right)} \frac{z^{\mathfrak{n}}}{n !},
\end{aligned}
$$

$\left(q, r, s \in \mathbb{N}_{0}, \quad|z|<1, \quad \mathfrak{R}\left(\gamma_{j}\right)>\mathfrak{R}\left(\alpha_{j}\right)>0, \quad(j=1, \cdots, q), \quad \min \{\mathfrak{R}(\mu), \mathfrak{R}(v)\}>0, \quad \mathfrak{R}(p) \geqq 0\right)$,

where, as usual, an empty product is interpreted as 1 and the involved parameters and the argument $z$ are tacitly assumed to be so constrained that the series on the right-hand side is absolutely convergent. The special case of the definition (1.13) when

$$
\mu=v=1, \quad \text { and } \quad q=r=s=1, \quad\left(a_{1}=1, \quad \alpha_{1}=b, \quad \gamma_{1}=c\right),
$$

coincides precisely with the definition (1.11). Also, for

$$
\mu=v=m, \quad \text { and } \quad q=r=s=1, \quad\left(a_{1}=1, \quad \alpha_{1}=b, \quad \gamma_{1}=c\right),
$$

and with the sequence $\left\{\kappa_{\ell}\right\}_{\ell \in \mathbb{N}_{0}}$ given by

$$
\mathrm{K}_{\ell}=\frac{(\rho)_{\ell}}{(\sigma)_{\ell}}, \quad\left(\ell \in \mathbb{N}_{0}\right),
$$

the definition (1.13) would obviously correspond to the Gauss type hypergeometric function introduced by Parmar [26, p. 44]:

$$
\begin{gathered}
\mathrm{F}_{p}^{(\rho, \sigma ; m)}(\mathrm{a}, \mathrm{b} ; \mathrm{c} ; z):=\sum_{n=0}^{\infty}(\mathrm{a})_{\mathrm{n}} \frac{\mathrm{B}^{(\rho, \sigma ; m)}(\mathrm{b}+\mathrm{n}, \mathrm{c}-\mathrm{b})}{\mathrm{B}(\mathrm{b}, \mathrm{c}-\mathrm{b})} \frac{z^{\mathrm{n}}}{\mathrm{n} !}, \\
(|z|<1, \quad \mathfrak{R}(\mathrm{p}) \geqq 0, \quad \min \{\mathfrak{R}(\rho), \mathfrak{R}(\sigma), \mathfrak{R}(\mathrm{m})\}>0, \quad \mathfrak{R}(\mathrm{c})>\mathfrak{R}(\mathrm{b})>0),
\end{gathered}
$$

which, in case

$$
\mu=v=m=1, \quad \text { and } \quad q=r=s=1, \quad\left(a_{1}=1, \quad \alpha_{1}=b, \quad \gamma_{1}=c\right),
$$

and with the sequence $\left\{\kappa_{\ell}\right\}_{\ell \in \mathbb{N}_{0}}$ given by

$$
\kappa_{\ell}=1, \quad\left(\ell \in \mathbb{N}_{0}\right),
$$

reduces immediately to the following $p$-Gauss hypergeometric function $F_{p}(a, b ; c ; z)$ studied by Chaudhry et al. [13]: 


$$
\begin{gathered}
F_{p}(a, b ; c ; z):=\sum_{n=0}^{\infty}(a)_{n} \frac{B(b+n, c-b ; p)}{B(b, c-b)} \frac{z^{n}}{n !}, \\
(p \geqq 0,|z|<1, \quad \Re(c)>\Re(b)>0) .
\end{gathered}
$$

Motivated essentially by the aforementioned and many other potential avenues of their applications, we propose to introduce and investigate here a new extension of the elliptic-type integrals, which is based upon the definition (1.12) of the generalized Beta function $\mathfrak{B}_{p ; \mu, v}^{\left(\left\{\kappa_{\ell}\right\}_{\ell \in \mathbb{N}_{0}}\right)}(\alpha, \beta)$. The extension proposed in this paper will be seen to be extremely useful. Many of the known properties of the elliptic-type integrals carry over naturally and simply for it. Furthermore, it provides connections with the complementary error function, the Whittaker function and the Meijer G-function as new representations for special parameter values of the extended elliptic-type integrals.

The plan of our paper is as follows: In Section 2, we introduce the generalized Appell and Lauricella type functions of two and more variables. In Section 3, we propose some generalizations of the incomplete and complete elliptic-type integrals. In Section 4, the extended complete elliptic-type integrals are presented in terms of the generalized hypergeometric type functions. In Section 5, various Mellin transform formulas are obtained for these extended elliptic-type integrals. In Section 6 several derivative and integral formulas are derived for the extended elliptic-type integrals. In Section 7, we express several special cases of these extended elliptic-type integrals in terms of some higher transcendental functions and give various infinite series representations containing the Whittaker function and the Laguerre polynomials and the products thereof. Finally, some concluding remarks and observations are presented in Section 8.

\section{Generalized Appell and Lauricella type functions}

In terms of the the generalized Beta type function $\mathfrak{B}_{p ; \mu, v}^{\left(\left\{\kappa_{\ell}\right\}_{\ell \in \mathbb{N}_{0}}\right)}(\alpha, \beta)$ is given by definition (1.12), we first introduce the generalized Appell and Lauricella type functions of two and $r$ variables as follows:

$$
\begin{aligned}
& \mathfrak{F}_{1}^{\left(\left\{\kappa_{\ell}\right\}_{\ell \in \mathbb{N}_{0} ; p ; \mu, v}\right)}\left(a, b, b^{\prime} ; c ; x, y\right):=\sum_{m, n=0}^{\infty}(b)_{m}\left(b^{\prime}\right)_{n} \frac{\mathfrak{B}_{p ; \mu, v}^{\left(\left\{\kappa_{\ell}\right\}_{\ell \in \mathbb{N}_{0}}\right)}(a+m+n, c-a)}{B(a, c-a)} \frac{x^{m}}{m !} \frac{y^{n}}{n !}, \\
& (\max \{|x|,|y|\}<1, \quad \mathfrak{R}(p) \geqq 0, \quad \min \{\mathfrak{R}(\mu), \mathfrak{R}(v)\}>0), \\
& \mathfrak{F}_{2}^{\left(\left\{\kappa_{\ell} \ell_{\ell \in \mathbb{N}_{0}} ; p ; \mu, v\right)\right.}\left(a, b, b^{\prime} ; c, c^{\prime} ; x, y\right) \\
& :=\sum_{m, n=0}^{\infty}(a)_{m+n} \frac{\mathfrak{B}_{p ; \mu, v}^{\left(\left\{\kappa_{\ell}\right\}_{\ell \in \mathbb{N}_{0}}\right)}(b+m, c-b) \mathfrak{B}_{p ; \mu, v}^{\left(\left\{\kappa_{\ell}\right\}_{\ell \in \mathbb{N}_{0}}\right)}\left(b^{\prime}+n, c^{\prime}-b^{\prime}\right)}{B(b, c-b) B\left(b^{\prime}, c^{\prime}-b^{\prime}\right)} \frac{x^{m}}{m !} \frac{y^{n}}{n !}, \\
& (|x|+|y|<1, \quad \mathfrak{R}(p) \geqq 0, \quad \min \{\mathfrak{R}(\mu), \mathfrak{R}(v)\}>0),
\end{aligned}
$$

and

$$
\begin{aligned}
\mathfrak{F}_{D,\left(\left\{k_{\ell}\right\}_{\ell \in \mathbb{N}_{0} ;} ; \mu ; \mu, v\right)}^{(r)}\left(a, b_{1}, \cdots, b_{r} ; c ; x_{1}, \cdots, x_{r}\right):= & \sum_{m_{1}, \cdots, m_{r}=0}^{\infty}\left(b_{1}\right)_{m_{1}} \cdots\left(b_{r}\right)_{m_{r}} \\
& \cdot \frac{\mathfrak{B}_{p ; \mu, v}^{\left(\{k \ell\} \in \mathbb{N}_{0}\right)}\left(a+m_{1}+\cdots+m_{r}, c-a\right)}{B(a, c-a)} \frac{x_{1}^{m_{1}}}{m_{1} !} \cdots \frac{x_{r}^{m_{r}}}{m_{r} !},
\end{aligned}
$$

$\left(\max \left\{\left|x_{1}\right|, \cdots,\left|x_{r}\right|\right\}<1, \quad \mathfrak{R}(p) \geqq 0, \quad \min \{\mathfrak{R}(\mu), \mathfrak{R}(v)\}>0\right)$. 
Theorem 2.1. The following integral representation for the generalized Appell type function in (2.1) holds true:

$$
\begin{aligned}
& \mathfrak{F}_{1}^{\left(\left\{\kappa_{\ell}\right\}_{\ell \in \mathbb{N}_{0} ;} ; p ; \mu, v\right)}\left(\mathrm{a}, \mathrm{b}, \mathrm{b}^{\prime} ; \mathrm{c} ; \mathrm{x}, \mathrm{y}\right)= \frac{\Gamma(\mathrm{c})}{\Gamma(\mathrm{a}) \Gamma(\mathrm{c}-\mathrm{a})} \int_{0}^{1} \mathrm{t}^{\mathrm{a}-1}(1-\mathrm{t})^{\mathrm{c}-\mathrm{a}-1}(1-x \mathrm{t})^{-\mathrm{b}}(1-\mathrm{yt})^{-\mathrm{b}^{\prime}} \\
& \cdot \Theta\left(\left\{\kappa_{\ell}\right\}_{\ell \in \mathbb{N}_{0}} ;-\frac{\mathrm{p}}{\mathrm{t}^{\mu}(1-\mathrm{t})^{v}}\right) \mathrm{dt}, \\
&(\mathfrak{R}(\mathrm{p})>0, \quad \mathrm{p}=0, \text { and } \max \{|\arg (1-x)|,|\arg (1-\mathrm{y})|\}<\pi, \quad \mathfrak{R}(\mathrm{c})>\mathfrak{R}(\mathrm{a})>0) .
\end{aligned}
$$

Proof. For convenience, we denote the second member of the assertion (2.4) by $\Lambda_{p}(x, y)$ and assume that $\max \{|x|,|y|\}<1$. Then, upon expressing

$$
(1-x t)^{-b}, \quad \text { and } \quad(1-y t)^{-b^{\prime}},
$$

as their Taylor-Maclaurin series, if we invert the order of summation and integration (which can easily be justified by absolute and uniform convergence), we find that

$$
\begin{aligned}
& \Lambda_{p}(x, y):= \frac{\Gamma(c)}{\Gamma(a) \Gamma(c-a)} \int_{0}^{1} t^{a-1}(1-t)^{c-a-1}(1-x t)^{-b}(1-y t)^{-b^{\prime}} \\
& \cdot \Theta\left(\left\{k_{\ell}\right\}_{\ell \in \mathbb{N}_{0}} ;-\frac{p}{t^{\mu}(1-t)^{v}}\right) d t \\
&= \frac{\Gamma(c)}{\Gamma(a) \Gamma(c-a)} \sum_{m, n=0}^{\infty}(b)_{m}\left(b^{\prime}\right)_{n} \frac{x^{m}}{m !} \frac{y^{n}}{n !} \\
& \cdot \int_{0}^{1} t^{a+m+n-1}(1-t)^{c-a-1} \Theta\left(\left\{k_{\ell}\right\}_{\ell \in \mathbb{N}_{0}} ;-\frac{p}{t^{\mu}(1-t)^{v}}\right) d t \\
& \quad(|x|+|y|<1, \quad \Re(c)>\Re(a)>0),
\end{aligned}
$$

which, in view of the definitions (1.12) and (2.1), yields the assertion (2.4) of Theorem 2.1.

Theorem 2.2. The following integral representation for the generalized Appell type function in (2.2) holds true:

$$
\begin{aligned}
& \mathfrak{F}_{2}^{\left(\left\{\kappa_{\ell}\right\}_{\ell \in \mathbb{N}_{0}} ; p ; \mu, v\right)}\left(a, b, b^{\prime} ; c, c^{\prime} ; x, y\right)=\frac{1}{B(b, c-b) B\left(b^{\prime}, c^{\prime}-b^{\prime}\right)} \\
& \cdot \int_{0}^{1} \int_{0}^{1} \frac{\mathrm{t}^{\mathrm{b}-1}(1-\mathrm{t})^{\mathrm{c}-\mathrm{b}-1} \mathrm{u}^{\mathrm{b}^{\prime}-1}(1-\mathrm{u})^{\mathrm{c}^{\prime}-\mathrm{b}^{\prime}-1}}{(1-x \mathrm{t}-\mathrm{yu})^{\mathrm{a}}} \\
& \cdot \Theta\left(\left\{\kappa_{\ell}\right\}_{\ell \in \mathbb{N}_{0}} ;-\frac{p}{t^{\mu}(1-t)^{v}}\right) \Theta\left(\left\{\kappa_{\ell}\right\}_{\ell \in \mathbb{N}_{0}} ;-\frac{p}{t^{\mu}(1-t)^{v}}\right) d t d u, \\
& \left(\mathfrak{R}(p)>0, \quad p=0, \text { and }|x|+|y|<1, \quad \mathfrak{R}(\mathrm{c})>\mathfrak{R}(\mathrm{b})>0, \quad \mathfrak{R}\left(\mathrm{c}^{\prime}\right)>\mathfrak{R}\left(\mathrm{b}^{\prime}\right)>0\right) .
\end{aligned}
$$

Proof. Since [32, p. 52, Eq. 1.6(2)]

$$
\sum_{m, n=0}^{\infty} f(m+n) \frac{x^{m}}{m !} \frac{y^{n}}{n !}=\sum_{N=0}^{\infty} f(N) \frac{(x+y)^{N}}{N !}
$$

it is easily seen that

$$
\begin{aligned}
(1-x t-y u)^{-a}= & \sum_{N=0}^{\infty} \frac{(a)_{N}}{N !}(x t+y u)^{N}=\sum_{m, n=0}^{\infty}(a)_{m+n} \frac{(x t)^{m}}{m !} \frac{(y u)^{n}}{n !}, \\
& (|x|+|y|<1, \quad \max \{|t|,|u|\}<1),
\end{aligned}
$$

which is rather instrumental in our demonstration of Theorem 2.2 along the lines of the proof of Theorem 2.1. 
Theorem 2.3. The following integral representation for the generalized Lauricella type function in (2.3) holds true:

$$
\begin{aligned}
& \mathfrak{F}_{\mathrm{D},\left(\left\{k_{\ell}\right\}_{\ell \in \mathbb{N}_{0}} ; p ; \mu, v\right)}^{(r)}\left(a, b_{1}, \cdots, b_{r} ; c ; x_{1}, \cdots, x_{r}\right)= \frac{\Gamma(c)}{\Gamma(a) \Gamma(c-a)} \\
& \cdot \int_{0}^{1} t^{a-1}(1-t)^{c-a-1}\left(1-x_{1} t\right)^{-b_{1}} \cdots\left(1-x_{r} t\right)^{-b_{r}} \\
& \cdot \Theta\left(\left\{k_{\ell}\right\}_{\ell \in \mathbb{N}_{0}} ;-\frac{p}{t^{\mu}(1-t)^{v}}\right) d t, \\
&\left(\mathfrak{R}(p)>0, \quad p=0, \text { and } \max \left\{\left|\arg \left(1-x_{1}\right)\right|, \cdots,\left|\arg \left(1-x_{r}\right)\right|\right\}<\pi, \quad \mathfrak{R}(c)>\Re(a)>0\right) .
\end{aligned}
$$

Proof. The proof of Theorem 2.3 is much akin to that of its special (two-variable) case (that is, Theorem 2.1 above) when $r=2$. We, therefore, omit the details involved.

\section{Generalized elliptic-type integrals}

In this section, we propose an extension of the classical incomplete and complete elliptic integrals of the first, second and third kind (with modulus $|k|$ and amplitude $\varphi$ ) as follows:

$$
\begin{aligned}
\mathcal{H}_{p ; \mu, \gamma}^{\left(\left\{k_{\ell}\right\}_{\ell \in \mathbb{N}_{0}}\right)}(\varphi, k, \gamma) & :=\int_{0}^{\varphi}\left(1-k^{2} \sin ^{2} \theta\right)^{\gamma-\frac{1}{2}} \Theta\left(\left\{k_{\ell}\right\}_{\ell \in \mathbb{N}_{0}} ;-\frac{p}{\sin ^{2 \mu} \theta \cos ^{2 \nu} \theta}\right) \mathrm{d} \theta \\
& =\int_{0}^{\sin \varphi} \frac{\left(1-k^{2} \mathrm{t}^{2}\right)^{\gamma-\frac{1}{2}}}{\sqrt{1-\mathrm{t}^{2}}} \Theta\left(\left\{k_{\ell}\right\}_{\ell \in \mathbb{N}_{0}} ;-\frac{p}{\mathrm{t}^{2 \mu}\left(1-\mathrm{t}^{2}\right)^{v}}\right) \mathrm{dt}, \\
(\mathfrak{R}(\mathrm{p}) & \left.>0, \quad \mathrm{p}=0, \text { and }\left|\mathrm{k}^{2}\right|<1, \quad 0 \leqq \varphi \leqq \frac{\pi}{2}\right),
\end{aligned}
$$

so that, obviously, in the special cases when

$$
\gamma=0, \quad \text { and } \quad \gamma=1,
$$

we have

$$
\begin{aligned}
& \mathcal{F}_{p ; \mu, \nu}^{\left(\left\{\kappa_{\ell}\right\}_{\ell \in \mathbb{N}_{0}}\right)}(\varphi, k):=\int_{0}^{\varphi} \frac{1}{\sqrt{1-k^{2} \sin ^{2} \theta}} \Theta\left(\left\{\kappa_{\ell}\right\}_{\ell \in \mathbb{N}_{0}} ;-\frac{p}{\sin ^{2 \mu} \theta \cos ^{2 v} \theta}\right) \mathrm{d} \theta \\
& =\int_{0}^{\sin \varphi} \frac{1}{\sqrt{\left(1-t^{2}\right)\left(1-k^{2} t^{2}\right)}} \Theta\left(\left\{\kappa_{\ell}\right\}_{\ell \in \mathbb{N}_{0}} ;-\frac{p}{t^{2 \mu}\left(1-t^{2}\right)^{v}}\right) d t, \\
& \left(\mathfrak{R}(\mathrm{p})>0, \quad p=0, \text { and }\left|k^{2}\right|<1, \quad 0 \leqq \varphi \leqq \frac{\pi}{2}\right) \text {, } \\
& \mathcal{E}_{p ; \mu, \nu}^{\left(\left\{\kappa_{\ell}\right\}_{\ell \in \mathbb{N}_{0}}\right)}(\varphi, k):=\int_{0}^{\varphi} \sqrt{1-k^{2} \sin ^{2} \theta} \Theta\left(\left\{\kappa_{\ell}\right\}_{\ell \in \mathbb{N}_{0}} ;-\frac{p}{\sin ^{2 \mu} \theta \cos ^{2 v} \theta}\right) \mathrm{d} \theta \\
& =\int_{0}^{\sin \varphi} \sqrt{\frac{1-\mathrm{k}^{2} \mathrm{t}^{2}}{1-\mathrm{t}^{2}}} \Theta\left(\left\{\kappa_{\ell}\right\}_{\ell \in \mathbb{N}_{0}} ;-\frac{p}{\mathrm{t}^{2 \mu}\left(1-\mathrm{t}^{2}\right)^{v}}\right) \mathrm{dt}, \\
& \left(\mathfrak{R}(p)>0, \quad p=0, \text { and }\left|k^{2}\right|<1, \quad 0 \leqq \varphi \leqq \frac{\pi}{2}\right) \text {, }
\end{aligned}
$$

and 


$$
\begin{aligned}
& \Pi_{p ; \mu, v}^{\left(\left\{\kappa_{\ell}\right\}_{\ell \in \mathbb{N}_{0}}\right)}\left(\varphi, \alpha^{2}, k\right):=\int_{0}^{\varphi} \frac{1}{\left(1-\alpha^{2} \sin ^{2} \theta\right) \sqrt{1-k^{2} \sin ^{2} \theta}} \\
& \cdot \Theta\left(\left\{\kappa_{\ell}\right\}_{\ell \in \mathbb{N}_{0}} ;-\frac{p}{\sin ^{2 \mu} \theta \cos ^{2 \nu} \theta}\right) \mathrm{d} \theta \\
& =\int_{0}^{\sin \varphi} \frac{1}{\left(1-\alpha^{2} t^{2}\right) \sqrt{\left(1-t^{2}\right)\left(1-k^{2} t^{2}\right)}} \\
& \cdot \Theta\left(\left\{\kappa_{\ell}\right\}_{\ell \in \mathbb{N}_{0}} ;-\frac{p}{t^{2 \mu}\left(1-t^{2}\right)^{v}}\right) d t \\
& \left(\mathfrak{R}(p)>0, \quad p=0 \text { and }\left|k^{2}\right|<1, \quad 0 \leqq \varphi \leqq \frac{\pi}{2}, \quad-\infty<\alpha^{2}<\infty\right) .
\end{aligned}
$$

In particular, when $\varphi=\frac{\pi}{2}$, these last equations (3.2), (3.3) and (3.4) reduce to the corresponding generalized complete elliptic-type integrals given by

$$
\begin{aligned}
\mathcal{H}_{p ; \mu, \nu}^{\left(\left\{\kappa_{\ell}\right\}_{\ell \in \mathbb{N}_{0}}\right)}(k, \gamma) & :=\int_{0}^{\frac{\pi}{2}}\left(1-k^{2} \sin ^{2} \theta\right)^{\gamma-\frac{1}{2}} \Theta\left(\left\{\kappa_{\ell}\right\}_{\ell \in \mathbb{N}_{0}} ;-\frac{p}{\sin ^{2 \mu} \theta \cos ^{2 \nu} \theta}\right) \mathrm{d} \theta \\
& =\int_{0}^{1} \frac{\left(1-\mathrm{k}^{2} \mathrm{t}^{2}\right)^{\gamma-\frac{1}{2}}}{\sqrt{1-\mathrm{t}^{2}}} \Theta\left(\left\{\kappa_{\ell}\right\}_{\ell \in \mathbb{N}_{0}} ;-\frac{p}{\mathrm{t}^{2 \mu}\left(1-\mathrm{t}^{2}\right)^{v}}\right) \mathrm{dt},
\end{aligned}
$$

so that, obviously, for

$$
\gamma=0, \quad \text { and } \quad \gamma=1
$$

we have

$$
\begin{aligned}
& \mathcal{K}_{p ; \mu, \nu}^{\left(\left\{k_{\ell}\right\}_{\ell \in \mathbb{N}_{0}}\right)}(k):=\int_{0}^{\frac{\pi}{2}} \frac{1}{\sqrt{1-k^{2} \sin ^{2} \theta}} \Theta\left(\left\{k_{\ell}\right\}_{\ell \in \mathbb{N}_{0}} ;-\frac{p}{\sin ^{2 \mu} \theta \cos ^{2 v} \theta}\right) \mathrm{d} \theta \\
& =\int_{0}^{1} \frac{1}{\sqrt{\left(1-t^{2}\right)\left(1-k^{2} t^{2}\right)}} \Theta\left(\left\{\kappa_{\ell}\right\}_{\ell \in \mathbb{N}_{0}} ;-\frac{p}{t^{2 \mu}\left(1-t^{2}\right)^{v}}\right) d t, \\
& \left(\mathfrak{R}(\mathrm{p})>0, \quad \mathrm{p}=0, \text { and }\left|k^{2}\right|<1\right) \text {, } \\
& \mathcal{E}_{p ; \mu, \nu}^{\left(\left\{\kappa_{\ell}\right\}_{\left.\ell \in \mathbb{N}_{0} ; \mu, v\right)}\right.}(k):=\int_{0}^{\frac{\pi}{2}} \sqrt{1-k^{2} \sin ^{2} \theta} \Theta\left(\left\{\kappa_{\ell}\right\}_{\ell \in \mathbb{N}_{0}} ;-\frac{p}{\sin ^{2 \mu} \theta \cos ^{2 v} \theta}\right) \mathrm{d} \theta \\
& =\int_{0}^{1} \sqrt{\frac{1-\mathrm{k}^{2} \mathrm{t}^{2}}{1-\mathrm{t}^{2}}} \Theta\left(\left\{\mathrm{k}_{\ell}\right\}_{\ell \in \mathbb{N}_{0}} ;-\frac{\mathrm{p}}{\mathrm{t}^{2 \mu}\left(1-\mathrm{t}^{2}\right)^{v}}\right) \mathrm{dt}, \\
& \left(\mathfrak{R}(\mathrm{p})>0, \quad p=0, \text { and }\left|k^{2}\right|<1\right) \text {, }
\end{aligned}
$$

and

$$
\begin{aligned}
\Pi_{p ; \mu, \nu}^{\left(\left\{k_{\ell}\right\}_{\ell \in \mathbb{N}_{0}}\right)}\left(\alpha^{2}, k\right):= & \int_{0}^{\frac{\pi}{2}} \frac{1}{\left(1-\alpha^{2} \sin ^{2} \theta\right) \sqrt{1-k^{2} \sin ^{2} \theta}} \\
& \cdot \Theta\left(\left\{k_{\ell}\right\}_{\ell \in \mathbb{N}_{0}} ;-\frac{p}{\sin ^{2 \mu} \theta \cos ^{2 v} \theta}\right) d \theta
\end{aligned}
$$




$$
\begin{aligned}
= & \int_{0}^{1} \frac{1}{\left(1-\alpha^{2} \mathrm{t}^{2}\right) \sqrt{\left(1-\mathrm{t}^{2}\right)\left(1-\mathrm{k}^{2} \mathrm{t}^{2}\right)}} \\
\cdot & \Theta\left(\left\{\kappa_{\ell}\right\}_{\ell \in \mathbb{N}_{0}} ;-\frac{p}{\mathrm{t}^{2 \mu}\left(1-\mathrm{t}^{2}\right)^{v}}\right) \mathrm{dt}, \\
(\mathfrak{R}(p)>0, \quad p & \left.=0, \text { and }\left|\mathrm{k}^{2}\right|<1, \quad \alpha^{2} \neq 1\right),
\end{aligned}
$$

respectively.

We now introduce the following generalization of the Epstein-Hubbell type elliptic-type integral in (1.7):

$$
\begin{aligned}
\Omega_{\gamma, p ; \mu, \nu}^{\left(\left\{\kappa_{\ell}\right\}_{\ell \in \mathbb{N}_{0}}\right)}(\kappa):= & \int_{0}^{\pi} \frac{1}{\left(1-\kappa^{2} \cos \theta\right)^{\gamma+\frac{1}{2}}} \Theta\left(\left\{\kappa_{\ell}\right\}_{\ell \in \mathbb{N}_{0}} ;-\frac{p}{\sin ^{2 \mu}\left(\frac{\theta}{2}\right) \cos ^{2 v}\left(\frac{\theta}{2}\right)}\right) \mathrm{d} \theta, \\
& \left(\mathfrak{R}(p)>0, \quad p=0, \text { and } 0 \leqq \kappa \leqq 1, \quad \gamma \in \mathbb{C}_{0}\right) .
\end{aligned}
$$

It is interesting to note that, if we put

$$
\mathrm{t}=\cos \left(\frac{\theta}{2}\right)=\sqrt{\frac{1+\cos \theta}{2}}
$$

in (3.5), we get the following relationship between the generalized elliptic-type integrals $\mathcal{H}_{\mathrm{p} ; \mu, \nu}^{\left(\left\{\kappa_{\ell}\right\}_{\ell \in \mathbb{N}_{0}}\right)}(k, \gamma)$ defined by (3.5) and $\Omega_{\gamma, p ; \mu, v}^{\left(\left\{\kappa_{\ell}\right\}_{\ell \in \mathbb{N}_{0}}\right)}(k)$ defined by (3.9):

$$
\mathcal{H}_{p ; \mu, \nu}^{\left(\left\{k_{\ell}\right\}_{\ell \in \mathbb{N}_{0}}\right)}(k, \gamma)=\frac{\left(2-k^{2}\right)^{\gamma-\frac{1}{2}}}{2^{\gamma+\frac{1}{2}}} \Omega_{-\gamma, p ; \gamma, \mu}^{\left(\left\{k_{\ell}\right\}_{\ell \in \mathbb{N}_{0}}\right)}\left(\frac{k}{\sqrt{2-k^{2}}}\right) .
$$

In terms of the complementary modulus $k^{\prime}$, the proposed extended complete elliptic integrals are defined by

$$
\mathcal{K}_{p ; \mu, v}^{\prime\left(\left\{k_{\ell}\right\}_{\ell \in \mathbb{N}_{0}}\right)}(k)=\mathcal{K}_{p ; \mu, v}^{\left(\left\{k_{\ell}\right\}_{\ell \in \mathbb{N}_{0}}\right)}\left(k^{\prime}\right)=\mathcal{K}_{p ; \mu, v}^{\left(\left\{k_{\ell}\right\}_{\ell \in \mathbb{N}_{0}}\right)}\left(\sqrt{1-k^{2}}\right), \quad\left(k^{\prime}:=\sqrt{1-k^{2}}\right),
$$

and

$$
\mathcal{E}_{p ; \mu, v}^{\prime\left(\left\{k_{\ell}\right\}_{\ell \in \mathbb{N}_{0}}\right)}(k)=\mathcal{E}_{p ; \mu, v}^{\left(\left\{k_{\ell}\right\}_{\ell \in \mathbb{N}_{0}}\right)}\left(k^{\prime}\right)=\mathcal{E}_{p ; \mu, v}^{\left(\left\{k_{\ell}\right\}_{\ell \in \mathbb{N}_{0}}\right)}\left(\sqrt{1-k^{2}}\right), \quad\left(k^{\prime}:=\sqrt{1-k^{2}}\right),
$$

respectively.

Remark 3.1. The special cases of (3.2) to (3.10) when $p=0$ or (alternatively) for

$$
\kappa_{\ell}=0, \quad(\ell \in \mathbb{N}),
$$

are easily seen to reduce to the classical incomplete and complete elliptic integrals (1.1) to (1.6), respectively (see, for details, [9], see also [8] and [19]).

\section{Connection with generalized hypergeometric functions}

In this section, we present generalized complete elliptic integrals in terms of generalized hypergeometric type functions of one, two and three variables.

Theorem 4.1. Let

$$
\mathfrak{R}(\mathrm{p})>0, \quad \text { and } \quad\left|k^{2}\right|<1 .
$$

Then 


$$
\begin{gathered}
\mathcal{H}_{p ; \mu, v}^{\left(\left\{k_{\ell}\right\}_{\ell \in \mathbb{N}_{0}}\right)}(k, \gamma)=\frac{\pi}{2}{ }_{2} \mathfrak{F}_{1}^{\left(\left\{\kappa_{\ell}\right\}_{\ell \in \mathbb{N}_{0}} ; p ; \mu, v\right)}\left(\frac{1}{2}-\gamma, \frac{1}{2} ; 1 ; k^{2}\right), \\
\mathcal{K}_{p ; \mu, v}^{\left(\left\{k_{\ell}\right\}_{\ell \in \mathbb{N}_{0}}\right)}(k)=\frac{\pi}{2}{ }_{2} \mathfrak{F}_{1}^{\left(\left\{k_{\ell}\right\}_{\ell \in \mathbb{N}_{0}} ; p ; \mu, v\right)}\left(\frac{1}{2}, \frac{1}{2} ; 1 ; k^{2}\right), \\
\mathcal{E}_{p ; \mu, v}^{\left(\left\{k_{\ell}\right\}_{\ell \in \mathbb{N}_{0}}\right)}(k)=\frac{\pi}{2}{ }_{2} \mathfrak{F}_{1}^{\left(\left\{k_{\ell}\right\}_{\ell \in \mathbb{N}_{0}} ; p ; \mu, v\right)}\left(-\frac{1}{2}, \frac{1}{2} ; 1 ; k^{2}\right),
\end{gathered}
$$

and

$$
\Omega_{\gamma, p ; \mu, \nu}^{\left(\left\{\kappa_{\ell}\right\}_{\ell \in \mathbb{N}_{0}}\right)}(\kappa):=\frac{\pi}{\left(1+\kappa^{2}\right)^{\gamma+\frac{1}{2}}} 2 \mathfrak{F}_{1}^{\left(\left\{\kappa_{\ell}\right\}_{\left.\ell \in \mathbb{N}_{0} ; p ; \mu, v\right)}\right.}\left(\gamma+\frac{1}{2}, \frac{1}{2} ; 1 ; \frac{2 \kappa^{2}}{1+\kappa^{2}}\right) .
$$

Proof. Letting $\mathrm{t}^{2}=\mathrm{u}$ in (3.5), (3.6) and (3.7) and using the definition (1.13), we get the desired relations (4.1), (4.2) and (4.3), respectively. Similarly, we can prove the relationship (4.4) asserted by Theorem 4.1.

Remark 4.2. In a special case when

$$
\mathrm{p}=0, \quad \text { and } \quad \mathrm{k}_{\ell}=0, \quad(\ell \in \mathbb{N}),
$$

equation (4.4) would reduce to a known result given by Weiss [36].

Theorem 4.3. For $\mathfrak{R}(\mathrm{p})>0$ and $\left|\mathrm{k}^{2}\right|<1$, each of the following relationships holds true:

$$
\begin{aligned}
& \mathcal{H}_{p ; \mu, v}^{\left(\left\{k_{\ell}\right\} \in \mathbb{N}_{0}\right)}(k, \gamma)=\frac{\pi}{2} \mathfrak{F}_{1}^{\left(\left\{k_{\ell}\right\} \ell \in \mathbb{N}_{0} ; p ; \mu, v\right)}\left(\frac{1}{2}, \frac{1}{2}, \frac{1}{2}-\gamma ; \frac{3}{2} ; 1, k^{2}\right), \\
& \mathcal{K}_{p ; \mu, \nu}^{\left(\left\{\kappa_{\ell}\right\}_{\ell \in \mathbb{N}_{0}}\right)}(k)=\frac{\pi}{2} \mathfrak{F}_{1}^{\left(\left\{\kappa_{\ell}\right\}_{\ell \in \mathbb{N}_{0}} ; p ; \mu, v\right)}\left(\frac{1}{2}, \frac{1}{2}, \frac{1}{2} ; \frac{3}{2} ; 1, k^{2}\right), \\
& \mathcal{E}_{p ; \mu, \nu}^{\left(\left\{\kappa_{\ell}\right\}_{\ell \in \mathbb{N}_{0}}\right)}(k)=\frac{\pi}{2} \mathfrak{F}_{1}^{\left(\left\{\kappa_{\ell}\right\}_{\ell \in \mathbb{N}_{0}} ; p ; \mu, v\right)}\left(\frac{1}{2}, \frac{1}{2},-\frac{1}{2} ; \frac{3}{2} ; 1, k^{2}\right), \\
& \Pi_{p}^{\left(\left\{k_{\ell}\right\}_{\ell \in \mathbb{N}_{0}}\right)}\left(\alpha^{2}, k\right)=\frac{\pi}{2} \mathfrak{F}_{1}^{\left(\left\{\kappa_{\ell}\right\}_{\ell \in \mathbb{N}_{0}} ; p ; \mu, v\right)}\left(\frac{1}{2}, 1, \frac{1}{2} ; 1 ; \alpha^{2}, k^{2}\right),
\end{aligned}
$$

and

$$
\Pi_{\mathcal{p} ; \mu, v}^{\left(\left\{\kappa_{\ell}\right\}_{\ell \in \mathbb{N}_{0}}\right)}\left(\alpha^{2}, k\right)=\frac{1}{4} \mathfrak{F}_{D,\left(\left\{k_{\ell}\right\}_{\ell \in \mathbb{N}_{0}} ; p ; \mu, v\right)}^{(3)}\left(\frac{1}{2}, \frac{1}{2}, \frac{1}{2}, \frac{1}{3} ; 1, \alpha^{2}, k^{2}\right) .
$$

Proof. Upon letting $\mathrm{t}^{2}=\mathrm{u}$ in the equations (3.5), (3.6), (3.7) and (3.8), if we make use of the integral representation in (2.4), we get the desired relations (4.5), (4.6), (4.7) and (4.8), respectively. On the other hand, by putting $t^{2}=u$ in (3.8) and using the integral representation (2.5) for $r=3$, we get the desired relation (4.9).

\section{A set of Mellin transform formulas}

The Mellin transform of a suitably integrable function $f(t)$ with index $s$ is defined, as usual, by

$$
\mathcal{M}\{f(\tau): \tau \rightarrow s\}:=\int_{0}^{\infty} \tau^{s-1} f(\tau) d \tau,
$$

whenever the improper integral in (5.1) exists (see, for details, [20] and [28]). 
Theorem 5.1. The following Mellin transform formula for $\left.\mathcal{H}_{p_{;} ; \mu, v}^{\left(\left\{\kappa_{\ell}\right\}_{\ell \in \mathbb{N}_{0}}\right.}\right)(\varphi, k, \gamma)$ in (3.1) holds true:

$$
\begin{aligned}
& \mathcal{M}\left\{\mathcal{H}_{p ; \mu, \gamma}^{\left(\left\{k_{\ell}\right\}_{\ell \in \mathbb{N}_{0}}\right)}(\varphi, k, \gamma): p \rightarrow s\right\}= \frac{\Gamma_{0}^{\left(\left\{k_{\ell}\right\}_{\ell \in \mathbb{N}_{0}}\right)}(s) \sin ^{2 \mu s+1} \varphi}{2\left(\mu s+\frac{1}{2}\right)} \\
& \cdot F_{1}\left(\mu s+\frac{1}{2}, \frac{1}{2}-\gamma, \frac{1}{2}-v s ; \mu s+\frac{3}{2} ; k^{2} \sin ^{2} \varphi, \sin ^{2} \varphi\right), \\
&\left(\mathfrak{R}(s)>0, \quad\left|k^{2}\right|<1, \quad 0 \leqq \varphi \leqq \frac{\pi}{2}\right),
\end{aligned}
$$

where $F_{1}$ denotes one of the four Appell's hypergeometric functions of two variables defined by (see, e.g., [31, p. 22, Eq. 1.3(2)]):

$$
F_{1}\left[\alpha, \beta_{1}, \beta_{2} ; \gamma ; x, y\right]=\sum_{m, n=0}^{\infty} \frac{(\alpha)_{m+n}\left(\beta_{1}\right)_{m}\left(\beta_{2}\right)_{n}}{(\gamma)_{m+n}} \frac{x^{m}}{m !} \frac{y^{n}}{n !}, \quad(\max \{|x|,|y|\}<1),
$$

Proof. Using the definition (5.1), we find from (3.1) that

$$
\begin{aligned}
& \mathcal{M}\left\{\mathcal{H}_{\mathfrak{p} ; \mu, \nu}^{\left(\left\{k_{\ell}\right\}_{\ell \in \mathbb{N}_{0}}\right)}(\varphi, k, \gamma): p \rightarrow s\right\}:=\int_{0}^{\infty} p^{s-1} \mathcal{H}_{\mathcal{p} ; \mu, \gamma}^{\left(\left\{k_{\ell}\right\}_{\ell \in \mathbb{N}_{0}}\right)}(\varphi, k, \gamma) \mathrm{dp} \\
& =\int_{0}^{\infty} p^{s-1}\left[\int_{0}^{\varphi}\left(1-k^{2} \sin ^{2} \theta\right)^{\gamma-\frac{1}{2}} \Theta\left(\left\{k_{\ell}\right\}_{\ell \in \mathbb{N}_{0}} ;-\frac{p}{\sin ^{2 \mu} \theta \cos ^{2 v} \theta}\right) d \theta\right] d p \\
& =\frac{1}{2} \int_{0}^{\infty} p^{s-1}\left[\int_{0}^{\sin ^{2} \varphi} \frac{\left(1-k^{2} t\right)^{\gamma-\frac{1}{2}}}{\sqrt{t(1-t)}} \Theta\left(\left\{\kappa_{\ell}\right\}_{\ell \in \mathbb{N}_{0}} ;-\frac{p}{t^{\mu}(1-t)^{v}}\right) d t\right] d p
\end{aligned}
$$

where we have also set

$$
\sin ^{2} \theta=\mathrm{t}, \quad \text { and } \quad \mathrm{d} \theta=\frac{\mathrm{dt}}{2 \sqrt{\mathrm{t}(1-\mathrm{t})}},
$$

in the inner $\theta$-integral. Upon interchanging the order of integration on the right-hand side, which can easily be justified by absolute convergence of the integrals involved under the constraints stated with Theorem 5.1, we get

$$
\begin{aligned}
& \left.\mathcal{M}\left\{\mathcal{H}_{\mathbf{p} ; \mu, v}^{\left(\left\{k_{\ell}\right\} \ell \in \mathbb{N}_{0}\right.}\right)(\varphi, k, \gamma): p \rightarrow s\right\} \\
& =\frac{1}{2} \int_{0}^{\sin ^{2} \varphi} \frac{\left(1-k^{2} t\right)^{\gamma-\frac{1}{2}}}{\sqrt{t(1-t)}}\left[\int_{0}^{\infty} p^{s-1} \Theta\left(\left\{k_{\ell}\right\}_{\ell \in \mathbb{N}_{0}} ;-\frac{p}{t^{\mu}(1-t)^{v}}\right) d p\right] d t \\
& =\frac{1}{2} \int_{0}^{\sin ^{2} \varphi} \mathrm{t}^{\mu s-\frac{1}{2}}(1-\mathrm{t})^{\gamma s-\frac{1}{2}}\left(1-\mathrm{k}^{2} \mathrm{t}\right)^{\gamma-\frac{1}{2}}\left(\int_{0}^{\infty} \omega^{s-1} \Theta\left(\left\{\kappa_{\ell}\right\}_{\ell \in \mathbb{N}_{0}} ;-\omega\right) \mathrm{d} \omega\right) \mathrm{dt},
\end{aligned}
$$

where we obviously have set

$$
\frac{p}{t^{\mu}(1-t)^{v}}=\omega, \quad \text { and } \quad d p=t^{\mu}(1-t)^{v} d \omega,
$$

in the inner $p$-integral. We now interpret the $\omega$-integral by means of the definition (1.9) (with $p=0$ ). We thus find that

$$
\mathcal{M}\left\{\mathcal{H}_{\mathrm{p} ; \mu, \nu}^{\left(\left\{k_{\ell}\right\}_{\ell \in \mathbb{N}_{0}}\right)}(\varphi, k, \gamma): p \rightarrow s\right\}=\frac{\Gamma_{0}^{\left(\left\{k_{\ell}\right\}_{\ell \in \mathbb{N}_{0}}\right)}(s)}{2} \int_{0}^{\sin ^{2} \varphi} \mathrm{t}^{\mu s-\frac{1}{2}}(1-\mathrm{t})^{\mu s-\frac{1}{2}}\left(1-k^{2} \mathrm{t}\right)^{\gamma-\frac{1}{2}} \mathrm{dt}
$$


which, upon setting

$$
\mathrm{t}=\left(\sin ^{2} \varphi\right) \tau, \quad \text { and } \quad \mathrm{dt}=\left(\sin ^{2} \varphi\right) \mathrm{d} \tau,
$$

yields

$$
\begin{aligned}
& \left.\mathcal{M}\left\{\mathcal{H}_{p ; \mu, \nu}^{\left(\left\{\kappa_{\ell}\right\}_{\ell \in \mathbb{N}_{0}}\right.}\right)(\varphi, k, \gamma): p \rightarrow s\right\}=\frac{\Gamma_{0}^{\left(\left\{\kappa_{\ell}\right\}_{\ell \in \mathbb{N}_{0}}\right)}(s) \sin ^{2 \mu s+1} \varphi}{2} \\
& \cdot \int_{0}^{1} \tau^{\mu s-\frac{1}{2}}\left(1-k^{2} \sin ^{2} \varphi \tau\right)^{\gamma-\frac{1}{2}}\left(1-\sin ^{2} \varphi \tau\right)^{v s-\frac{1}{2}} \mathrm{~d} \tau .
\end{aligned}
$$
[5]):

Finally, by using the following integral representation (see, e.g., [31, p. 276, Eq. 9.4(7)], see also [4] and

$$
\begin{gathered}
F_{1}\left(a, b, b^{\prime} ; c ; x, y\right)=\frac{\Gamma(c)}{\Gamma(a) \Gamma(c-a)} \int_{0}^{1} t^{a-1}(1-t)^{c-a-1}(1-x t)^{-b}(1-y t)^{-b^{\prime}} d t, \\
(\max \{|\arg (1-x)|,|\arg (1-y)|\}<\pi, \quad \mathfrak{R}(c)>\Re(a)>0),
\end{gathered}
$$

we get the desired Mellin transform formula (5.2) asserted by Theorem 5.1.

Theorem 5.2. The following Mellin transform formula for $\Pi_{p ; \mu, v}^{\left(\left\{\kappa_{\ell}\right\}_{\ell \in \mathbb{N}_{0}}\right)}\left(\varphi, \alpha^{2}, k\right)$ in (3.4) holds true:

$$
\begin{aligned}
& \mathcal{M}\left\{\Pi_{p ; \mu, v}^{\left(\left\{k_{\ell}\right\}_{\ell \in \mathbb{N}_{0}}\right)}\left(\varphi, \alpha^{2}, k\right): p \rightarrow s\right\}= \frac{\Gamma_{0}^{\left(\left\{k_{\ell}\right\}_{\ell \in \mathbb{N}_{0}}\right)}(s) \sin ^{2 \mu s+1} \varphi}{2\left(\mu s+\frac{1}{2}\right)} \\
& \cdot F_{\mathrm{D}}^{(3)}\left(\mu s+\frac{1}{2}, 1, \frac{1}{2}, \frac{1}{2}-v s ; \mu s+\frac{3}{2} ; \alpha^{2} \sin ^{2} \varphi, k^{2} \sin ^{2} \varphi, \sin ^{2} \varphi\right), \\
&\left(\mathfrak{R}(s)>0, \quad\left|k^{2}\right|<1, \quad 0 \leqq \varphi \leqq \frac{\pi}{2}, \quad-\infty<\alpha^{2}<\infty\right) .
\end{aligned}
$$

Proof. The proof of Theorem 5.2 runs parallel to that of Theorem 5.1. It similarly makes use of the following integral representation (see, e.g., [31, p. 283, Eq. 9.4(34) (with $n=3)$ ]):

$$
\begin{aligned}
& \mathrm{F}_{\mathrm{D}}^{(3)}\left(\mathrm{a}, \mathrm{b}_{1}, \mathrm{~b}_{2}, \mathrm{~b}_{3} ; c ; x, y, z\right)= \frac{\Gamma(\mathrm{c})}{\Gamma(\mathrm{a}) \Gamma(\mathrm{c}-\mathrm{a})} \\
& \cdot \int_{0}^{1} \mathrm{t}^{\mathrm{a}-1}(1-\mathrm{t})^{\mathrm{c}-\mathrm{a}-1}(1-x \mathrm{t})^{-\mathrm{b}_{1}}(1-y \mathrm{t})^{-\mathrm{b}_{2}}(1-z \mathrm{t})^{-\mathrm{b}_{3}} \mathrm{dt}, \\
&\left.\left(\max _{\{}|\arg (1-x)|,|\arg (1-y)|,|\arg (1-z)|\right\}<\pi, \quad \mathfrak{R}(\mathrm{c})>\mathfrak{R}(\mathrm{a})>0\right) .
\end{aligned}
$$

The details involved may be omitted.

Remark 5.3. The Appell functions $F_{1}$ is expressible in terms of the Kampé de Fériet's and the SrivastavaDaoust hypergeometric functions in two variables (see, e.g., [31, p. 22, Eq. 1.3 (2)] and [31, p. 37, Eq. 1.4 (21)]):

$$
F_{1}\left[\alpha, \beta_{1}, \beta_{2} ; \gamma ; x, y\right]=F_{1: 0 ; 0}^{1: 1 ; 1}\left[\begin{array}{c}
\alpha: \beta_{1} ; \beta_{2} ; \\
\gamma: \ldots ; \ldots ;
\end{array}\right]
$$

and 


$$
F_{1}\left[\alpha, \beta_{1}, \beta_{2} ; \gamma ; x, y\right]=F_{1: 0 ; 0}^{1: 1 ; 1}\left[\begin{array}{l}
(\alpha: 1,1):\left(\beta_{1}, 1\right) ;\left(\beta_{1}, 1\right) ; \\
(\gamma: 1,1): \_; y
\end{array}\right] .
$$

Now, by first applying the relationship (5.4) in (5.2) and the relationship (5.5) in (5.2) and then using the Legendre duplication formula for the Gamma function (see, e.g., [31, p. 17, Eq. 1.2(14)]):

$$
\Gamma(2 z)=\frac{2^{2 z-1}}{\sqrt{\pi}} \Gamma(z) \Gamma\left(z+\frac{1}{2}\right)
$$

we can deduce interesting Mellin transform formulas for $\mathcal{H}_{p ; \mu, \gamma}^{\left(\left\{\kappa_{\ell}\right\}_{\ell \in \mathbb{N}_{0}}\right)}(\varphi, k, \gamma)$ in (3.1) as asserted by Corollary 5.4 below. Further, if take $\gamma=0$ and $\gamma=1$, we can deduce certain interesting Mellin transform formulas for

$$
\mathcal{F}_{p ; \mu, v}^{\left(\left\{\kappa_{\ell}\right\}_{\ell \in \mathbb{N}_{0}}\right)}(\varphi, k), \quad \text { and } \quad \mathcal{E}_{p ; \mu, \nu}^{\left(\left\{\kappa_{\ell}\right\}_{\ell \in \mathbb{N}_{0}}\right)}(\varphi, k),
$$

in (3.2) and (3.3) as asserted by Corollary 5.5 below. The proofs of Corollaries 5.4 and 5.5 will be omitted here.

Corollary 5.4. Each of the following Mellin transform formulas holds true:

$$
\begin{aligned}
& \mathcal{M}\left\{\mathcal{H}_{p ; \mu, v}^{\left(\left\{\kappa_{\ell}\right\}_{\ell \in \mathbb{N}_{0}}\right)}(\varphi, k, \gamma): p \rightarrow s\right\}=\frac{\Gamma_{0}^{\left(\left\{\kappa_{\ell}\right\}_{\ell \in \mathbb{N}_{0}}\right)}(s) \sin ^{2 \mu s+1} \varphi}{2\left(\mu s+\frac{1}{2}\right)} \\
& \cdot F_{1: 0 ; 0}^{1: 1 ; 1}\left[\begin{array}{c}
\mu s+\frac{1}{2}: \frac{1}{2}-\gamma ; \frac{1}{2}-v s ; \\
\mu s+\frac{3}{2}: \longrightarrow ; k^{2} \sin ^{2} \varphi, \sin ^{2} \varphi
\end{array}\right] \text {, }
\end{aligned}
$$

and

$$
\begin{aligned}
& \mathcal{M}\left\{\mathcal{H}_{p ; \mu, v}^{\left(\left\{\kappa_{\ell}\right\}_{\ell \in \mathbb{N}_{0}}\right)}(\varphi, k, \gamma): p \rightarrow s\right\}=\frac{\Gamma_{0}^{\left(\left\{\kappa_{\ell}\right\}_{\ell \in \mathbb{N}_{0}}\right)}(s) \sin ^{2 \mu s+1} \varphi}{2\left(\mu s+\frac{1}{2}\right)} \\
& \cdot F_{1: 0 ; 0}^{1: 1 ; 1}\left[\begin{array}{l}
(2 \mu s+1: 2,2):\left(\frac{1}{2}-\gamma, 1\right) ;\left(\frac{1}{2}-v s, 1\right) ; \\
(2 \mu s+2: 2,2):-; k^{2} \sin ^{2} \varphi, \sin ^{2} \varphi
\end{array}\right] \text {. }
\end{aligned}
$$

Corollary 5.5. Each of the following Mellin transform formulas for

$$
\left.\mathcal{F}_{p ; \mu, v}^{\left(\left\{k_{\ell}\right\}_{\ell \in \mathbb{N}_{0}}\right)}(\varphi, k), \quad \text { and } \quad \mathcal{E}_{p ; \mu, v}^{\left(\left\{k_{\ell}\right\} \ell \in \mathbb{N}_{0}\right.}\right)(\varphi, k),
$$

in (3.2) and (3.3) holds true:

$$
\begin{aligned}
& \mathcal{M}\left\{\mathcal{F}_{\mathcal{p} ; \mu, v}^{\left(\left\{\kappa_{\ell}\right\}_{\ell \in \mathbb{N}_{0}}\right)}(\varphi, k): p \rightarrow s\right\}=\frac{\Gamma_{0}^{\left(\left\{\kappa_{\ell}\right\}_{\ell \in \mathbb{N}_{0}}\right)}(\mathrm{s}) \sin ^{2 \mu s+1} \varphi}{2(\mu \mathrm{s}+1 / 2)} \\
& \cdot F_{1}\left(\mu s+\frac{1}{2}, \frac{1}{2}, \frac{1}{2}-v s ; \mu s+\frac{3}{2} ; k^{2} \sin ^{2} \varphi, \sin ^{2} \varphi\right), \\
& \mathcal{M}\left\{\mathcal{F}_{p ; \mu, v}^{\left(\left\{\kappa_{\ell}\right\}_{\ell \in \mathbb{N}_{0}}\right)}(\varphi, k): p \rightarrow s\right\}=\frac{\Gamma_{0}^{\left(\left\{\kappa_{\ell}\right\}_{\ell \in \mathbb{N}_{0}}\right)}(s) \sin ^{2 \mu s+1} \varphi}{2(\mu s+1 / 2)}
\end{aligned}
$$




$$
\begin{aligned}
& \cdot F_{1: 0 ; 0}^{1: 1 ; 1}\left[\begin{array}{c}
\mu s+\frac{1}{2}: \frac{1}{2} ; \frac{1}{2}-v s ; \\
\mu s+\frac{1}{2}:-;-;
\end{array} k^{2} \sin ^{2} \varphi, \sin ^{2} \varphi\right] \\
& \left.\mathcal{M}\left\{\mathcal{F}_{p ; \mu, v}^{\left(\left\{\kappa_{\ell}\right\} \ell \in \mathbb{N}_{0}\right.}\right)(\varphi, k): p \rightarrow s\right\}=\frac{\Gamma_{0}^{\left(\left\{k_{\ell}\right\} \ell \in \mathbb{N}_{0}\right)}(s) \sin ^{2 \mu s+1} \varphi}{2\left(\mu s+\frac{1}{2}\right)} \\
& \cdot F_{1: 0 ; 0}^{1: 1 ; 1}\left[\begin{array}{c}
(2 \mu s+1: 2,2):\left(\frac{1}{2}, 1\right) ;\left(\frac{1}{2}-v s, 1\right) ; \\
(2 \mu s+2: 2,2):-; k^{2} \sin ^{2} \varphi, \sin ^{2} \varphi
\end{array}\right] \text {, } \\
& \left.\mathcal{M}\left\{\mathcal{E}_{\mathrm{p} ; \mu, v}^{\left(\left\{k_{\ell}\right\} \ell \in \mathbb{N}_{0}\right.}\right)(\varphi, k): p \rightarrow s\right\}=\frac{\left.\Gamma_{0}^{\left(\left\{k_{\ell}\right\} \ell \in \mathbb{N}_{0}\right.}\right)(s) \sin ^{2 \mu s+1} \varphi}{2\left(\mu s+\frac{1}{2}\right)} \\
& \cdot F_{1}\left(\mu s+\frac{1}{2},-\frac{1}{2}, \frac{1}{2}-v s ; \mu s+\frac{3}{2} ; k^{2} \sin ^{2} \varphi, \sin ^{2} \varphi\right), \\
& \left.\mathcal{M}\left\{\mathcal{E}_{p ; \mu, v}^{\left(\left\{\kappa_{\ell}\right\}_{\ell \in \mathbb{N}_{0}}\right.}\right)(\varphi, k): p \rightarrow s\right\}=\frac{\Gamma_{0}^{\left(\left\{\kappa_{\ell}\right\}_{\ell \in \mathbb{N}_{0}}\right)}(s) \sin ^{2 \mu s+1} \varphi}{2\left(\mu s+\frac{1}{2}\right)} \\
& \cdot F_{1: 0 ; 0}^{1: 1 ; 1}\left[\begin{array}{c}
\mu s+\frac{1}{2}:-\frac{1}{2} ; \frac{1}{2}-v s ; \\
\mu s+\frac{3}{2}:-;-;
\end{array} k^{2} \sin ^{2} \varphi, \sin ^{2} \varphi\right]
\end{aligned}
$$

and

$$
\begin{aligned}
& \mathcal{M}\left\{\mathcal{E}_{\mathrm{p} ; \mu, v}^{\left(\left\{\kappa_{\ell}\right\}_{\ell \in \mathbb{N}_{0}}\right)}(\varphi, \mathrm{k}): \mathrm{p} \rightarrow s\right\}=\frac{\Gamma_{0}^{\left(\left\{k_{\ell}\right\}_{\ell \in \mathbb{N}_{0}}\right)}(s) \sin ^{2 \mu s+1} \varphi}{2\left(\mu \mathrm{s}+\frac{1}{2}\right)}
\end{aligned}
$$

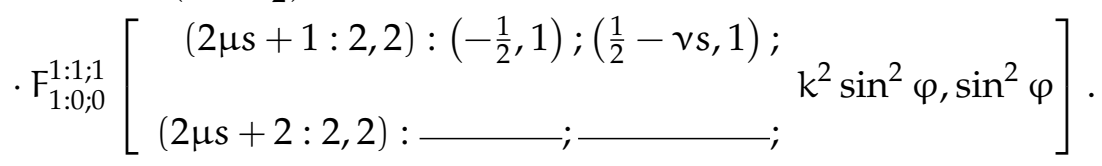

Theorem 5.6. Each of the following Mellin transform formulas for

$$
\mathcal{H}_{p ; \mu, v}^{\left(\left\{\kappa_{\ell}\right\}_{\ell \in \mathbb{N}_{0}}\right)}(k, \gamma), \quad \mathcal{K}_{p ; \mu, \nu}^{\left(\left\{\kappa_{\ell}\right\}_{\ell \in \mathbb{N}_{0}}\right)}(k), \quad \text { and } \quad \mathcal{E}_{p ; \mu, \nu}^{\left(\left\{\kappa_{\ell}\right\}_{\ell \in \mathbb{N}_{0}}\right)}(k),
$$

in (3.5), (3.6) and (3.7) holds true:

$$
\begin{aligned}
& \mathcal{M}\left\{\mathcal{H}_{\mathrm{p} ; \mu, v}^{\left(\left\{\kappa_{\ell}\right\}_{\ell \in \mathbb{N}_{0}}\right)}(k, \gamma): p \rightarrow s\right\}=\frac{\Gamma_{0}^{\left(\left\{\kappa_{\ell}\right\}_{\ell \in \mathbb{N}_{0}}\right)}(\mathrm{s}) \mathrm{B}\left(\mu \mathrm{s}+\frac{1}{2}, v s+\frac{1}{2}\right)}{2} \\
& \cdot_{2} F_{1}\left(\mu s+\frac{1}{2}, \frac{1}{2}-\gamma ; \mu s+v s+1 ; k^{2}\right), \\
& \left(\mathfrak{R}(s)>0, \quad\left|k^{2}\right|<1, \quad 0 \leqq \varphi \leqq \frac{\pi}{2}\right), \\
& \mathcal{M}\left\{\mathcal{K}_{\mathrm{p} ; \mu, v}^{\left(\left\{\kappa_{\ell}\right\}_{\ell \in \mathbb{N}_{0}}\right)}(\mathrm{k}): p \rightarrow s\right\}=\frac{\Gamma_{0}^{\left(\left\{\kappa_{\ell}\right\}_{\ell \in \mathbb{N}_{0}}\right)}(\mathrm{s}) \mathrm{B}\left(\mu \mathrm{s}+\frac{1}{2}, v s+\frac{1}{2}\right)}{2}
\end{aligned}
$$




$$
\begin{array}{r}
\cdot_{2} F_{1}\left(\mu s+\frac{1}{2}, \frac{1}{2} ; \mu s+v s+1 ; k^{2}\right), \\
\left(\mathfrak{R}(s)>0, \quad\left|k^{2}\right|<1, \quad 0 \leqq \varphi \leqq \frac{\pi}{2}\right),
\end{array}
$$

and

$$
\begin{gathered}
\mathcal{M}\left\{\mathcal{E}_{p ; \mu, v}^{\left(\left\{\kappa_{\ell \ell \ell \in \mathbb{N}_{0}}\right)\right.}(k): p \rightarrow s\right\}=\frac{\Gamma_{0}^{\left(\left\{\kappa_{\ell}\right\}_{\ell \in \mathbb{N}_{0}}\right)}(\mathrm{s}) \mathrm{B}\left(\mu \mathrm{s}+\frac{1}{2}, v s+\frac{1}{2}\right)}{2} \\
\cdot{ }_{2} \mathrm{~F}_{1}\left(\mu \mathrm{s}+\frac{1}{2},-\frac{1}{2} ; \mu \mathrm{s}+v s+1 ; k^{2}\right), \\
\left(\mathfrak{R}(\mathrm{s})>0, \quad\left|\mathrm{k}^{2}\right|<1, \quad 0 \leqq \varphi \leqq \frac{\pi}{2}\right) .
\end{gathered}
$$

Proof. By putting $\varphi=\frac{\pi}{2}$ in (5.2), (5.6) and (5.7) and then using the following identity (see [20, p. 239, Eq. (10)]):

$$
\mathrm{F}_{1}\left[\alpha, \beta_{1}, \beta_{2} ; \gamma ; x, 1\right]=\frac{\Gamma(\gamma) \Gamma\left(\gamma-\alpha-\beta_{2}\right)}{\Gamma(\gamma-\alpha) \Gamma\left(\gamma-\beta_{2}\right)}{ }_{2} F_{1}\left(\alpha, \beta_{1} ; \gamma-\beta_{2} ; x\right),
$$

we get the desired Mellin transform formulas asserted by Theorem 5.6.

Theorem 5.7. The following Mellin transform formula for $\prod_{p ; \mu, v}^{\left(\{k \in\} \in \mathbb{N}_{0}\right)}\left(\alpha^{2}, k\right)$ in (3.8) holds true:

$$
\begin{aligned}
& \mathcal{M}\left\{\Pi_{p ; \mu, v}^{\left(\left\{k_{\ell}\right\}_{\ell \in \mathbb{N}_{0}}\right)}\left(\alpha^{2}, \mathrm{k}\right): p \rightarrow s\right\}= \frac{1}{2} \Gamma_{0}^{\left(\left\{\mathrm{k}_{\ell}\right\}_{\ell \in \mathbb{N}_{0}}\right)}(\mathrm{s}) \mathrm{B}\left(\mu \mathrm{s}+\frac{1}{2}, v s+\frac{1}{2}\right) \\
& \cdot \mathrm{F}_{1}\left(\mu \mathrm{s}+\frac{1}{2}, 1, \frac{1}{2} ; \mu \mathrm{s}+v s+1 ; \alpha^{2}, \mathrm{k}^{2}\right), \\
&\left(\mathfrak{R}(\mathrm{s})>0, \text { and }\left|\mathrm{k}^{2}\right|<1, \quad-\infty<\alpha^{2}<\infty\right) .
\end{aligned}
$$

Proof. Putting $\varphi=\frac{\pi}{2}$ in (5.3) and using the following easily derivable reduction formula:

$$
F_{D}^{(3)}\left(\alpha, \beta_{1}, \beta_{2}, \beta_{3} ; \gamma ; x, y, 1\right)=\frac{\Gamma(\gamma) \Gamma\left(\gamma-\alpha-\beta_{3}\right)}{\Gamma(\gamma-\alpha) \Gamma\left(\gamma-\beta_{3}\right)} F_{1}\left[\alpha, \beta_{1}, \beta_{2} ; \gamma-\beta_{3} ; x, y\right],
$$

we get the desired result (5.10) asserted by Theorem 5.7.

Remark 5.8. If we choose the sequence $\left\{\kappa_{\ell}\right\}_{\ell \in \mathbb{N}_{0}}$ as in (1.14) and set $\mu=\nu=1$ in the assertions (5.8) and (5.9) of Theorem 5.6 and in the assertion (5.10) of Theorem 5.7, we obtain the corresponding Mellin transform formulas for the extended complete elliptic integrals as asserted by Corollary 5.9.

Corollary 5.9. Each of the following Mellin transform formulas holds true:

$$
\begin{gathered}
\mathcal{M}\left\{\mathrm{K}_{\mathrm{p}}(\mathrm{k}): \mathrm{p} \rightarrow \mathrm{s}\right\}=\frac{\sqrt{\pi} \Gamma\left(\mathrm{s}+\frac{1}{2}\right)}{2^{2 s+1} \mathrm{~s}}{ }_{2} \mathrm{~F}_{1}\left(\frac{1}{2}, \mathrm{~s}+\frac{1}{2} ; 2 \mathrm{~s}+1 ; \mathrm{k}^{2}\right), \\
\mathcal{M}\left\{\mathrm{E}_{\mathrm{p}}(\mathrm{k}): \mathrm{p} \rightarrow \mathrm{s}\right\}=\frac{\sqrt{\pi} \Gamma\left(\mathrm{s}+\frac{1}{2}\right)}{2^{2 s+1} \mathrm{~s}} \mathrm{~F}_{1}\left(-\frac{1}{2}, \mathrm{~s}+\frac{1}{2} ; 2 \mathrm{~s}+1 ; \mathrm{k}^{2}\right),
\end{gathered}
$$

and

$$
\mathcal{M}\left\{\Pi_{p}\left(\alpha^{2}, k\right): p \rightarrow s\right\}=\frac{\sqrt{\pi} \Gamma\left(s+\frac{1}{2}\right)}{2^{2 s+1} s} F_{1}\left(s+\frac{1}{2}, 1, \frac{1}{2} ; 2 s+1 ; \alpha^{2}, k^{2}\right)
$$


Remark 5.10. If we set $s=1$ in (5.11) and (5.12), we get the following interesting relationships between the original and the extended complete elliptic integrals:

$$
\int_{0}^{\infty} K_{p}(k) d p=\frac{\pi}{16}{ }_{2} F_{1}\left(\frac{1}{2}, \frac{1}{2} ; 3 ; k^{2}\right)
$$

which, in light of the known result [27, p. 473, Entry (93)], yields

$$
\int_{0}^{\infty} K_{p}(k) d p=\frac{1}{3 k^{2}}\left[K(k)-\left(2-k^{2}\right) D(k)\right]
$$

and

$$
\int_{0}^{\infty} E_{p}(k) d p=\frac{\pi}{16}{ }_{2} F_{1}\left(-\frac{1}{2}, \frac{3}{2} ; 3 ; k^{2}\right),
$$

which, by means of the known result [27, p. 469, Entry (20)], can be put in the form:

$$
\int_{0}^{\infty} E_{p}(k) d p=\frac{1}{15 k^{2}}\left[\left(1+k^{2}\right) K(k)-2\left(1-k^{2}+k^{4}\right) D(k)\right] .
$$

\section{Derivative and integral formulas}

In this section, we state (without proof) several derivative and integrals formulas for the generalized elliptic-type integrals.

Theorem 6.1. Each of the following derivative formulas holds true:

$$
\begin{aligned}
& \frac{\mathrm{d}}{\mathrm{dk}}\left\{\mathcal{E}_{\mathrm{p} ; \mu, v}^{\left(\left\{k_{\ell}\right\}_{\ell \in \mathbb{N}_{0}}\right)}(\varphi, \mathrm{k})\right\}=\frac{1}{\mathrm{k}}\left[\mathcal{E}_{\mathrm{p} ; \mu, \nu}^{\left(\left\{\kappa_{\ell}\right\}_{\ell \in \mathbb{N}_{0}}\right)}(\varphi, k)-\mathcal{F}_{p ; \mu, \nu}^{\left(\left\{\kappa_{\ell}\right\}_{\ell \in \mathbb{N}_{0}}\right)}(\varphi, k)\right], \\
& \frac{\mathrm{d}}{\mathrm{dk}}\left\{\mathcal{E}_{p ; \mu, v}^{\left(\left\{k_{\ell}\right\}_{\ell \in \mathbb{N}_{0}}\right)}\right\}=\frac{1}{\mathrm{k}}\left[\mathcal{E}_{p ; \mu, v}^{\left(\left\{k_{\ell}\right\}_{\ell \in \mathbb{N}_{0}}\right)}-\mathcal{K}_{p ; \mu, v}^{\left(\left\{k_{\ell}\right\}_{\ell \in \mathbb{N}_{0}}\right)}\right],
\end{aligned}
$$

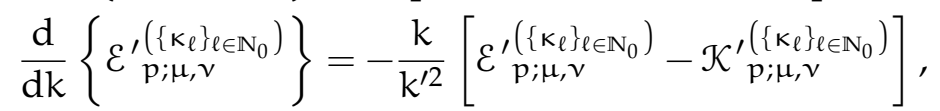

$$
\begin{aligned}
& \left.\frac{\mathrm{d}}{\mathrm{d} \mathrm{k}^{\prime}}\left\{\mathcal{E}_{\mathrm{p} ; \mu, v}^{\left(\left\{\mathrm{k}_{\ell}\right\}_{\ell \in \mathbb{N}_{0}}\right)}\right\}=-\frac{\mathrm{k}^{\prime}}{\mathrm{k}^{2}}\left[\mathcal{E}_{\mathrm{p} ; \mu, v}^{\left(\left\{k_{\ell}\right\} \ell \in \mathbb{N}_{0}\right.}\right)-\mathcal{K}_{\mathrm{p} ; \mu, v}^{\left(\left\{k_{\ell}\right\}_{\ell \in \mathbb{N}_{0}}\right)}\right], \\
& \frac{\mathrm{d}^{\mathrm{m}}}{\mathrm{d}\left(\mathrm{k}^{2}\right)^{\mathrm{m}}}\left\{\mathcal{K}_{\mathrm{p} ; \mu, v}^{\left(\left\{k_{\ell}\right\}_{\ell \in \mathbb{N}_{0}}\right)}\right\}=\frac{\pi[(2 \mathrm{~m}) !]^{2}}{2(\mathrm{~m} !)^{3} 16^{\mathrm{m}}} 2 \mathfrak{F}_{1}^{\left(\left\{k_{\ell}\right\}_{\ell \in \mathbb{N}_{0}} ; p ; \mu, v\right)}\left(\frac{1}{2}+\mathrm{m}, \frac{1}{2}+\mathrm{m}, 1+\mathrm{m} ; \mathrm{k}^{2}\right), \\
& \frac{\mathrm{d}^{\mathrm{m}}}{\mathrm{d}\left(\mathrm{k}^{2}\right)^{\mathrm{m}}}\left\{\mathcal{E}_{\mathrm{p} ; \mu, v}^{\left(\left\{\mathrm{k}_{\ell}\right\}_{\ell \in \mathbb{N}_{0}}\right)}\right\}=\frac{\pi}{2} \frac{\left(\frac{1}{2}\right)_{\mathrm{m}}\left(-\frac{1}{2}\right)_{\mathrm{m}}}{\mathrm{m} !} 2 \mathfrak{F}_{1}^{\left(\left\{\kappa_{\ell \ell}\right\}_{\ell \in \mathbb{N}_{0}} ; p ; \mu, v\right)}\left(-\frac{1}{2}+\mathrm{m}, \frac{1}{2}+\mathrm{m}, 1+\mathrm{m} ; \mathrm{k}^{2}\right), \\
& \frac{\partial^{n}}{\partial p^{n}}\left\{\mathcal{K}_{p ; \mu, v}^{\left(\left\{\kappa_{\ell}\right\}_{\ell \in \mathbb{N}_{0}}\right)}(k)\right\}=(-1)^{n} \frac{\pi}{2}{ }_{2} \mathfrak{F}_{1}^{\left(\left\{\kappa_{\ell}\right\}_{\ell \in \mathbb{N}_{0}} ; p ; \mu, v\right)}\left(\frac{1}{2}, \frac{1}{2}-n ; 1-2 n ; k^{2}\right),
\end{aligned}
$$

and

$$
\frac{\partial^{n}}{\partial p^{n}}\left\{\mathcal{E}_{p ; \mu, v}^{\left(\left\{k_{\ell}\right\}_{\ell \in \mathbb{N}_{0}}\right)}(k)\right\}=(-1)^{n} \frac{\pi}{2}{ }^{2} \mathfrak{F}_{1}^{\left(\left\{k_{\ell}\right\}_{\ell \in \mathbb{N}_{0}} ; p ; \mu, v\right)}\left(-\frac{1}{2}, \frac{1}{2}-n ; 1-2 n ; k^{2}\right) .
$$

Theorem 6.2. Each of the following integral formulas holds true:

$$
\int\left[\mathcal{K}_{p ; \mu, v}^{\left(\left\{\kappa_{\ell}\right\}_{\ell \in \mathbb{N}_{0}}\right)}-\varepsilon_{p ; \mu, v}^{\left(\left\{\kappa_{\ell}\right\}_{\ell \in \mathbb{N}_{0}}\right)}\right] \frac{\mathrm{dk}}{\mathrm{k}}=-\mathcal{E}_{p ; \mu, v}^{\left(\left\{\kappa_{\ell}\right\}_{\ell \in \mathbb{N}_{0}}\right)}
$$




$$
\begin{aligned}
& \left.\int \mathcal{K}_{p ; \mu, v}^{\left(\left\{\kappa_{\ell}\right\}_{\ell \in \mathbb{N}_{0}}\right)} \frac{\mathrm{dk}}{\mathrm{k}^{2}}=-\frac{1}{\mathrm{k}} \mathcal{E}_{\mathrm{p} ; \mu, v}^{\left(\left\{\kappa_{\ell}\right\} \ell \in \mathbb{N}_{0}\right.}\right),
\end{aligned}
$$

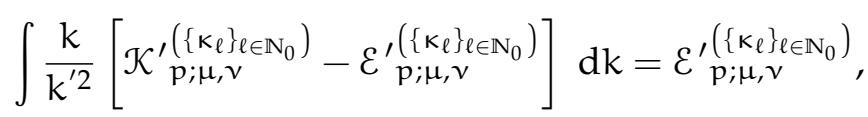

and

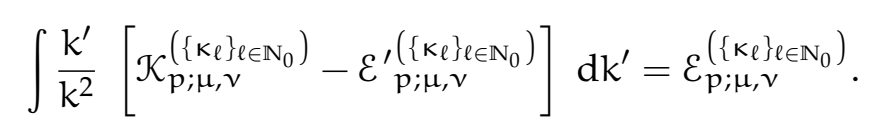

\section{Special values and connections with other special functions}

In this section, we first find the special values of $K_{p}(k), K_{p}^{\prime}(k), E_{p}(k)$ and $E_{p}^{\prime}(k)$.

Theorem 7.1. Each of the following relations holds true:

$$
\begin{aligned}
& K_{p}(0)=K_{p}^{\prime}(1)=\frac{1}{2} B\left(\frac{1}{2}, \frac{1}{2} ; p\right), \\
& E_{p}(0)=E_{p}^{\prime}(1)=\frac{1}{2} B\left(\frac{1}{2}, \frac{1}{2} ; p\right), \\
& E_{p}(1)=E_{p}^{\prime}(0)=\frac{1}{2} B\left(\frac{1}{2}, 1 ; p\right),
\end{aligned}
$$

and

$$
K_{p}(1)=K_{p}^{\prime}(0)=\frac{1}{2} B\left(\frac{1}{2}, 0 ; p\right) .
$$

Remark 7.2. The Meijer G-function [16, p. 232, Eq. (5.124)], the Whittaker function $W_{\kappa, \mu}(z)[16$, p. 229, Eq. (5.107)], the complementary error function $\operatorname{erfc}(z)[16$, p. 229, Eq. (5.106)] and the confluent hypergeometric function $U(a, b, z)[16$, p. 229, Eq. (5.109)] are expressible in terms of the extended Beta function $\mathrm{B}(\alpha, \beta ; p)$ for $\mathfrak{R}(p)>0$ as follows:

$$
\begin{aligned}
B(x, y ; p) & =\sqrt{\pi} 2^{1-x-y} G_{2,3}^{3,0}\left(4 p \mid \begin{array}{c}
\frac{x+y}{2}, \frac{x+y+1}{2} \\
0, x, y
\end{array}\right), \\
B\left(\frac{1}{2}, \frac{1}{2} ; p\right) & =\sqrt{\frac{\pi}{2}} p^{-\frac{1}{4}} e^{-2 p} W_{-\frac{1}{4}, \frac{1}{4}}(4 p), \\
B\left(\frac{1}{2}, \frac{1}{2} ; p\right) & =\sqrt{\pi} \operatorname{erfc}(2 \sqrt{p}),
\end{aligned}
$$

and

$$
\text { B }\left(\frac{1}{2}, \frac{1}{2} ; p\right)=\sqrt{\pi} e^{-4 p} u\left(\frac{1}{2}, \frac{1}{2} ; 4 p\right) .
$$

Now, by applying the relationships (7.1) to (7.2) in Theorem 6.2, we can deduce several interesting representations of the extended complete elliptic integrals. These are given (without proof) in Corollary 7.3 below.

Corollary 7.3. Each of the following representations holds true:

$$
\mathrm{K}_{\mathrm{p}}(0)=\mathrm{K}_{\mathrm{p}}^{\prime}(1)=\mathrm{E}_{\mathrm{p}}(0)=\mathrm{E}_{\mathrm{p}}^{\prime}(1)=\frac{\pi}{2} \operatorname{erfc}(2 \sqrt{\mathrm{p}}),
$$




$$
\begin{aligned}
& K_{p}(0)=K_{p}^{\prime}(1)=E_{p}(0)=E_{p}^{\prime}(1)=\sqrt{\pi} p^{-\frac{1}{4}} 2^{-\frac{3}{2}} e^{-2 p} W_{-\frac{1}{4}, \frac{1}{4}}(4 p), \\
& K_{p}(0)=K_{p}^{\prime}(1)=E_{p}(0)=E_{p}^{\prime}(1)=\frac{\sqrt{\pi}}{2} G_{1,2}^{2,0}\left(\begin{array}{c|c}
4 p & 1 \\
0, \frac{1}{2}
\end{array}\right), \\
& E_{p}(1)=E_{p}^{\prime}(0)=\frac{1}{2} \sqrt{\frac{\pi}{2}} G_{2,3}^{3,0}\left(\begin{array}{l|c}
4 p & \frac{3}{4}, \frac{5}{4} \\
0, \frac{1}{2}, 1
\end{array}\right),
\end{aligned}
$$

and

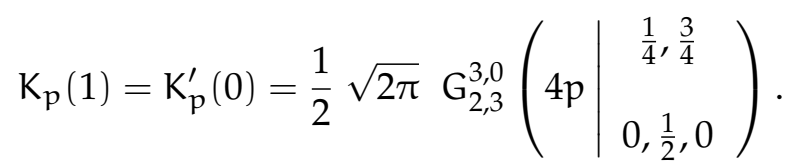

Next, in terms of the simple Laguerre polynomials $L_{n}(x)$ given by (see, e.g., [16, p. 238, Eq. (5.152)])

$$
L_{n}(x):=L_{n}^{(0)}(x), \quad \text { and } \quad L_{n}^{(\alpha)}(x):=\sum_{j=0}^{n}\left(\begin{array}{c}
n+\alpha \\
n-j
\end{array}\right) \frac{(-x)^{j}}{j !}
$$

we derive the representations asserted by Theorem 7.4 below.

Theorem 7.4. Each of the following Laguerre polynomial representations holds true:

$$
\begin{aligned}
& K_{p}(k)=\frac{\pi}{2} e^{-2 p} \sum_{m, n=0}^{\infty} \frac{\left(\frac{1}{2}\right)_{m+1}\left(\frac{1}{2}\right)_{n+1}}{(m+n+2) !} L_{m}(p) L_{n}(p)_{2} F_{1}\left(\frac{1}{2}, m+\frac{3}{2} ; m+n+3 ; k^{2}\right), \\
& E_{p}(k)=\frac{\pi}{2} e^{-2 p} \sum_{m, n=0}^{\infty} \frac{\left(\frac{1}{2}\right)_{m+1}\left(\frac{1}{2}\right)_{n+1}}{(m+n+2) !} L_{m}(p) L_{n}(p)_{2} F_{1}\left(-\frac{1}{2}, m+\frac{3}{2} ; m+n+3 ; k^{2}\right),
\end{aligned}
$$

and

$$
\begin{aligned}
\Pi_{p}\left(\alpha^{2}, k\right)= & \frac{\pi}{2} e^{-2 p} \sum_{m, n=0}^{\infty} \frac{\left(\frac{1}{2}\right)_{m+1}\left(\frac{1}{2}\right)_{n+1}}{(m+n+2) !} L_{m}(p) L_{n}(p) \\
& \cdot F_{1}\left(m+\frac{3}{2}, 1, \frac{1}{2} ; m+n+3 ; \alpha^{2}, k^{2}\right)
\end{aligned}
$$

Proof. Upon setting

$$
\sin ^{2} \theta=t, \quad \text { and } \quad d \theta=\frac{d t}{2 \sqrt{t(1-t)}}
$$

in (3.6), we get

$$
K_{p}(k)=\frac{1}{2} \int_{0}^{1} t^{-\frac{1}{2}}(1-t)^{-\frac{1}{2}}\left(1-k^{2} t\right)^{-\frac{1}{2}} \exp \left(-\frac{p}{t(1-t)}\right) d t .
$$

We now make use of the known identity for the simple Laguerre polynomials (see, e.g., [16, p. 238, Eq. (5.155)], see also [25]):

$$
\exp \left(-\frac{p}{t(1-t)}\right)=e^{-2 p} \sum_{m, n=0}^{\infty} L_{m}(p) L_{n}(p) t^{m+1}(1-t)^{n+1},
$$


in the integral representation (7.6). After a little simplification, we get the desired representation (7.3).

A similar procedure for $E_{p}(k)$ and $\Pi_{p}\left(\alpha^{2}, k\right)$ yields (7.4) and (7.5).

Theorem 7.5. Each of the following Laguerre polynomial and Whittaker function representations holds true:

$$
\begin{aligned}
K_{p}(k)= & \frac{1}{4 \sqrt{\pi}} \exp \left(-\frac{3 p}{2}\right) \sum_{m, n=0}^{\infty}\left(\frac{1}{2}\right)_{n} p^{\frac{2 m+2 n-1}{4}} \\
& \cdot L_{m}(p) W_{-\frac{1}{4}(2 m+2 n+5), \frac{1}{4}(2 m+2 n+1)}(p) \frac{k^{2 n}}{n !}
\end{aligned}
$$

and

$$
\begin{aligned}
E_{p}(k)= & \frac{1}{4 \sqrt{\pi}} \exp \left(-\frac{3 p}{2}\right) \sum_{m, n=0}^{\infty}\left(-\frac{1}{2}\right)_{n} p^{\frac{2 m+2 n-1}{4}} \\
& \cdot L_{m}(p) W_{-\frac{1}{4}(2 m+2 n+5), \frac{1}{4}(2 m+2 n+1)}(p) \frac{k^{2 n}}{n !} .
\end{aligned}
$$

Proof. Using the following known identity for the Laguerre polynomials (see, e.g., [16, p. 239]):

$$
\exp \left(-\frac{p}{t(1-t)}\right)=(1-t) \exp \left(-\frac{p(1+t)}{t}\right) \sum_{m=0}^{\infty} L_{m}(p) t^{m}
$$

in the integral representation (7.6), we get

$$
\begin{aligned}
E_{p}(k)= & \frac{1}{2} \int_{0}^{1} t^{-\frac{1}{2}}(1-t)^{-\frac{1}{2}} \\
& \cdot\left(\sum_{n=0}^{\infty}\left(-\frac{1}{2}\right)_{n} \frac{\left(t k^{2}\right)^{n}}{n !}\right)\left[(1-t) \exp \left(-\frac{p(1+t)}{t}\right) \sum_{m=0}^{\infty} L_{m}(p) t^{m}\right] d t
\end{aligned}
$$

Upon interchanging the order of summations and integration, we find from (7.9) that

$$
E_{p}(k)=\frac{1}{2} e^{-p} \sum_{m, n=0}^{\infty}\left(-\frac{1}{2}\right)_{n} L_{m}(p)\left[\int_{0}^{1} t^{n+m-\frac{1}{2}}(1-t)^{\frac{1}{2}} \exp \left(-\frac{p}{t}\right) d t\right] \frac{\left(k^{2}\right)^{n}}{n !} .
$$

Finally, by using the integral representation [16, p. 362, Eq. 3.471(2)]:

$$
\begin{gathered}
\int_{0}^{1} t^{\mu-1}(1-t)^{v-1} \exp \left(-\frac{p}{t}\right) d t=p^{\frac{\mu-1}{2}} \exp \left(-\frac{p}{2}\right) \Gamma(v) W_{\frac{1-\mu-2 v}{2}, \frac{\mu}{2}}(p), \\
(\mathfrak{R}(v)>0, \quad \mathfrak{R}(p)>0),
\end{gathered}
$$

in (7.10), we arrive at the desired representation (7.7).

A similar procedure for $E_{p}(k)$ would yield the second assertion (7.8) of Theorem 7.5.

\section{Concluding remarks and observations}

Our present investigation is motivated essentially by many potential avenues of applications of various families of incomplete and complete elliptic-type integrals as well as the generalized Beta function $\mathfrak{B}_{p ; \mu, v}^{\left(\left\{\kappa_{\ell}\right\}_{\ell \in \mathbb{N}_{0}}\right)}(\alpha, \beta)$ defined by (1.12). By means of the definition (1.12), we have introduced and systematically studied new extensions of the generalized Appell and Lauricella type functions of two and more 
variables and also of the incomplete and complete elliptic-type integrals. We have shown that the extensions proposed in this paper are potentially useful and that many of the known properties of the elliptic-type integrals carry over naturally and simply in terms of these extensions. We have also provided connections with the complementary error function, the Whittaker function and the G-function as new representations for special parameter values of the extended elliptic-type integral.

\section{References}

[1] M. Abramowitz, I. A. Stegun (Eds.), Handbook of Mathematical Functions, with Formulas, Graphs, and Mathematical Tables, Third printing, with corrections, National Bureau of Standards Applied Mathematics Series, Superintendent of Documents, U.S. Government Printing Office, Washington, D.C., (1965). 1

[2] M. Ali Özarslan, E. Özergin, Some generating relations for extended hypergeometric functions via generalized fractional derivative operator, Math. Comput. Modelling, 52 (2010), 1825-1833. 1

[3] L. C. Andrews, Special Functions for Engineers and Applied Mathematicians, Macmillan Company, New York, (1985). 1

[4] P. Appell, J. Kampé de Fériet, Fonctions Hypergéomtriques et Hypersphériques; Polynômes d'Hermite, Gauthier-Villars, Paris, (1926). 5

[5] W. N. Bailey, Generalized hypergeometric Series, Cambridge Tracts in Mathematics and Mathematical Physics, Vol. 32, Stechert-Hafner, Incorporated, New York, (1964). 5

[6] G. Barton, Do attractive scattering potential concentrate particles at the origin in one, two and three dimensions? III: High energies in quantum mechanics, Proc. Roy. Soc. London Sect. A: Math. Phys. Eng. Sci., 388 (1983), 445-456. 1

[7] M. J. Berger, J. C. Lamkin, Sample calculations of gamma-ray penetration into shelters: Contributions of sky shine and roof contamination, J. Res. Nat. Bur. Standards Sect. B, 60 (1958), 109-116. 1

[8] P. J. Bushell, On a generalization of Barton's integral and related integrals of complete elliptic integrals, Math. Proc. Cambridge Philos. Soc., 101 (1987), 1-5. 1, 3.1

[9] P. F. Byrd, M. D. Friedman, Handbook of Elliptic Integrals for Engineers and Scientists, Second edition, revised, Die Grundlehren der mathematischen Wissenschaften, Band 67, Springer-Verlag, Berlin-Heidelberg-New Yok, (1971). $1,3.1$

[10] B. C. Carlson, Some series and bounds for incomplete elliptic integrals, J. Math. and Phys., 40 (1961), 125-134. 1

[11] B. C. Carlson, Special Functions of Applied Mathematics, Academic Press [Harcourt Brace Jovanovich, Publishers], New York-London, (1977). 1

[12] M. A. Chaudhry, A. Qadir, M. Raflqu, S. M. Zubair, Extension of Euler's beta function, J. Comput. Appl. Math., 78 (1997), 19-32. 1

[13] M. A. Chaudhry, A. Qadir, H. M. Srivastava, R. B. Paris, Extended hypergeometric and confluent hypergeometric functions, Appl. Math. Comput., 159 (2004), 589-602. 1

[14] M. A. Chaudhry, S. M. Zubair, Generalized incomplete gamma functions with applications, J. Comput. Appl. Math., 55 (1994), 99-124.

[15] M. A. Chaudhry, S. M. Zubair, Extended incomplete gamma functions with applications, J. Math. Anal. Appl., 274 (2002), 725-745.

[16] M. A. Chaudhry, S. M. Zubair, On a Class of Incomplete Gamma Functions with Applications, Chapman \& Hall/CRC, Boca Raton, FL, (2002). 1, 7.2, 7, 7, 7, 7

[17] D. Cvijović, J. Klinowski, On the integration of incomplete elliptic integrals, Proc. Roy. Soc. London Ser. A, 444 (1994), 525-532. 1

[18] D. Cvijović, J. Klinowski, Integrals involving complete elliptic integrals, J. Comput. Appl. Math., 106 (1999), $169-175$. 1

[19] L. F. Epstein, J. H. Hubbell, Evaluation of a generalized elliptic-type integral, J. Res. Nat. Bur. Standards Sect. B, 67 (1963), 1-17. 1, 3.1

[20] A. Erdélyi, W. Magnus, F. Oberhettinger, F. G. Tricomi, Higher Transcendental Functions, Vols. I and II (Based, in part, on notes left by Harry Bateman), McGraw-Hill Book Company, Inc., New York-Toronto-London, (1953). 5, 5

[21] E. L. Kaplan, Multiple elliptic integrals, J. Math. Phys., 29 (1950), 69-75. 1

[22] S.-D. Lin, L.-F. Chang, H. M. Srivastava, A certain class of incomplete elliptic integrals and associated definite integrals, Appl. Math. Comput., 215 (2009), 1176-1184. 1

[23] S.-D. Lin, H. M. Srivastava, J.-C. Yao, Some classes of generating relations associated with a family of the generalized Gauss type hypergeometric functions, Appl. Math. Inform. Sci., 9 (2015), 1731-1738. 1, 1

[24] M.-J. Luo, R. K. Raina, Extended generalized hypergeometric functions and their applications, Bull. Math. Anal. Appl., 5 (2013), 65-77. 1

[25] A. R. Miller, Remarks on a generalized beta function, J. Comput. Appl. Math., 100 (1998), 23-32. 7

[26] R. K. Parmar, A new generalization of gamma, beta, hypergeometric and confluent hypergeometric functions, Matematiche (Catania), 68 (2013), 33-52. 1 
[27] A. P. Prudnikov, Y. A. Brychkov, O. I. Marichev, Integrals and Series, Vol. 3, More Special Functions, Translated from the Russian by G. G. Gould, Gordon and Breach Science Publishers, New York, (1990). 5.10

[28] I. N. Sneddon, The Use of Integral Transforms, McGraw-Hill, New York, (1972). 5

[29] H. M. Srivastava, Some elliptic integrals of Barton and Bushell, J. Phys. A: Math. Gen., 28 (1995), 2305-2312. 1

[30] H. M. Srivastava, S. Bromberg, Some families of generalized elliptic-type integrals, Math. Comput. Modelling, 21 (1995), 29-38. 1

[31] H. M. Srivastava, P. W. Karlsson, Multiple Gaussian Hypergeometric Series, Ellis Horwood Series: Mathematics and Its Applications, Ellis Horwood Limited, Chichester; Halsted Press [John Wiley \& Sons, Incorporated], New York, (1985). 5.1, 5, 5, 5.3, 5

[32] H. M. Srivastava, H. L. Manocha, A Treatise on Generating Functions, Ellis Horwood Series: Mathematics and its Applications, Ellis Horwood Limited, Chichester; Halsted Press [John Wiley \& Sons, Incorporated], New York, (1984). 2

[33] H. M. Srivastava, R. K. Parmar, P. Chopra, A class of extended fractional derivative operators and associated generating relations involving hypergeometric functions, Axioms, 1 (2012), 238-258. 1, 1

[34] R. Srivastava, A note on the Epstein-Hubbell generalized elliptic-type integral, Appl. Math. Comput., 69 (1995), $255-262$. 1

[35] H. M. Srivastava, R. N. Siddiqi, A unified presentation of certain families of elliptic-type integrals related to radiation field problems, Radiat. Phys. Chem., 46 (1995), 303-315. 1

[36] G. H. Weiss, A note on a generalized elliptic integral, J. Res. Nat. Bur. Standards Sect. B, 68 (1964), 1-2. 1, 4.2 